\title{
STABILITY IN DYNAMICAL SYSTEMS I
}

\author{
E. D. Courant \\ Brookhaven National Laboratory \\ Upton, New York 11979 \\ R. D. RUTH, W. T. WENG \\ Stanford Linear Accelerator Center \\ Stanford University, Stanford, California, 9 \$305
}

\section{TABLE OF CONTENTS}

1. Introduction. . . . . . . . . . . . . . 3

2. Hamiltonian Dynamics . . . . . . . . . . . . . . 6

_- 2.1 Equations of Motion . . . . . . . . . . . . . . 6

2.2 Symmetry, Integrals, and Cyclic Coordinates . . . . . 7

2.3 Motion Near a Known Periodic Solution . . . . . . . . . 8

2.4 Change of Independent Variable . . . . . . . . . . . . . 9

2.5 The Motion of a Particle in an Accelerator . . . . . . . . 10

2.6 Floquet's Theorem . . . . . . . . . . . . . . . . 13

2.7 Higher-Order Terms . . . . . . . . . . . . . . 15

3. Canonical Transformations . . . . . . . . . . . . 16

3.1 The Generating Function of a Canonical Transformation . . 17

3.2 Action-Angle Variables for the Harmonic Oscillator . . . . 19

3.3 Deviation from a Known Solution . . . . . . . . . . . 20

4. Linear Equations with Periodic Coefficients . . . . . . . . . . 22

4.1 The Matrix Approach . . . . . . . . . . . . . 23

4.2 The Phase-Amplitude Form of the Solution. . . . . . . . 26

4.3. Action-Angle Variables . . . . . . . . . . . . . . . . 29

${ }^{*}$ Work supported by the Department of Energy, contract DE - AC03 - 76SF00515

Invited paper presented at the $3^{\text {rd }}$ Summer School on

High Energy Particle Accelerators, Upton, New York, July 6-16, 1983. 
5. Canonical Perturbation Theory ........... 32

6. One-Dimensional Cubic Nonlinear Resonances . . . . . . . . . 37

6.1 The Behavior Far From Resonance . . . . . . . . . 37

6.2 The Third Integer Resonance . . . . . . . . . . . . 39

6.3 Phase Space Structure . . . . . . . . . . . . . . . 41

6.4 Stopband Width of the Third-Order Resonance . . . . . 42

7. A One-Dimensional Isolated Nonlinear Resonance . . . . . . . 43

7.1 Fixed Points . . . . . . . . . . . . . 43

7.2 Resonance Island Width . . . . . . . . . . . 45

7.3 Island Separation . . . . . . . . . . . . . . . 46

7.4 The Pendulum Equation . . . . . . . . . 47

7.5 The Distinction of Resonances 3, 4,5 or Higher . . . . . 48

_. 7.6 Solution on the Separatrix . . . . . . . . . . 49

8. Summary . . . . . . . . . . . . . 50 50

9. Bibliography ................. 51 


\title{
STABILITY IN DYNAMICAL SYSTEMS I
}

\author{
E. D. COURANT \\ Brookhaven National Laboratory \\ Upton, New York 11973 \\ R. D. RUTH, W. T. WENG \\ Stanford Linear Accelerator Center \\ Stanford University, Stanford, California, 94905
}

\section{INTRODUCTION}

A dynamical system is a collection of objects subject to some law of force. This leads to a set of differential equations which govern the motion. In this paper we are interested in those sets of differential equations which are derivable from a Hamiltonian using Hamilton's equations. That is, if we are given coordinates $q$ and 'canonical' momenta $p$, together with a Hamiltonian function $H(q, p, t)$, then the differential equations governing the motion are given by

$$
\frac{d q}{d t}=\frac{\partial H}{\partial p} \quad, \quad \frac{d p}{d t}=-\frac{\partial H}{\partial q} .
$$

Note that the above relations are valid for systems of $n$ dimensions provided that we interpret $q$ and $p$ as $n$-dimensional vectors. It is useful to view the system as the motion of a point in the $2 n$-dimensional space $(q, p)$ called phase space. We refer to a point in this space with the symbol $X$. Then the motion of a particle subject to Hamilton's equations can be viewed as a transformation of this point in phase space. The questions are how and where it moves and what are the characteristics of its motion. If the Hamiltonian is independent of the time $t$ (conservation of energy), then the motion is restricted to a $(2 n-1)$-dimensional surface in $2 n$-dimensional space on which $H=E$. What else may happen?

(a) Equilibrium. In this case a particular point in phase space does not move under the equations of motion. This is a fixed point of the transformation in phase space. Motion starting there remains there; however, points close to a fixed point may or may not remain close to the fixed point.

(b) Periodic motion. In this case an orbit exists that returns from a point $X_{0}$ back to the same point $X_{0}$ after a finite time $T$. The transformation

$$
X(t)=M(t) X(0)
$$


in phase space can be considered a mapping of phase space onto itself. If a particular orbit $X_{1}(t)$ is periodic with period $T$, then the mapping $M(T)$ has a fixed point at the position $X_{1}(0)$. If initial conditions near this point stay 'near' forever, the fixed point is 'stable'; if they blow up exponentially, it is unstable. Interestingly enough, it turns out that there is sometimes an intermediate case in which orbits stay near for a very long time, but not forever (diffusion). Note also that if $M(T)$ has fixed points, then $M(T)^{n}$ also has these fixed points and possibly others which repeat after $2,3, \ldots, n$ cycles of the original one.

(c) Closed surfaces. If a function $F(q, p, t)$ remains constant, it is an integral' of the motion. One such integral for a time independent Hamiltonian is the energy $E$ mentioned above. All motion occurs on a surface with $H(q, p)=E$. For other integrals there are other functions of $q, p$, and $t$ which are constant. A system is called integrable if there are $n$ independent integrals of the motion. In many cases these confine the motion to a closed $n$-dimensional surface thus guaranteeing stability.

(d) Nonintegrable Systems. There are many systems for which $n$ independent integrals do not exist. These are called nonintegrable. To illustrate this consider a system of two degrees of freedom with the Hamiltonian

$$
H_{0}=\frac{1}{2}\left(p_{x}^{2}+p_{y}^{2}\right)+\frac{1}{2}\left(a x^{2}+2 b x y+a y^{2}\right) \text {. }
$$

This is just a pair of coupled harmonic oscillators. For this system there are two independent integrals given by

$$
\begin{aligned}
& W_{1}=\left(p_{x}+p_{y}\right)^{2}+(a+b)(x+y)^{2} \\
& W_{2}=\left(p_{x}-p_{y}\right)^{2}+(a-b)(x-y)^{2} .
\end{aligned}
$$

That is, $W_{1}$ and $W_{2}$ are constant as $\left(x, p_{x}, y, p_{y}\right)$ evolve according to Hamilton's Equations with the Hamiltonian in Eq. (1.3). Now we add a nonlinear term to $H$

$$
H=H_{0}+\epsilon\left(x^{3}-3 x y^{2}\right)
$$

where $\epsilon$ is small, and ask if there are still two invariants given by $W_{1}$ and $W_{2}$ with small terms added.

This is not, in general, the case, and the actual situation is much more complicated although it appears to be typical of nonintegrable systems. At small enough amplitudes, the system acts as if a second integral exists, and as if the motion is confined to a two-dimensional subspace of the three-dimensional energy surface. At larger amplitudes the motion ranges 'ergodically' over all of the emergy surface except for that part taken up by the 'small' motions 
mentioned above. Mathematically it can be proved that such behavior exists, and this behavior can be demonstrated quite easily by computation.

However, in the case just discussed it is not necessary for the second integral to exist in order to have stability. The two-dimensional example with a time independent Hamiltonian is equivalent to a one-dimensional problem with a time dependent Hamiltonian. In this case we ask if one integral exists. If it does not, we ask if there are closed invariant curves in phase space. If these curves exist, then points inside must stay inside giving stability. For nonintegrable systems these curves do exist while integrals do not. The existence of these curves is the subject of a theorem called the KAM theorem (Kolmogorov, Arnold, Moser). ${ }^{4}$

(e) Arnold Diffusion. For three or more dimensions, or two or more dimensions with a time dependent Hamiltonian, it may happen that extra integrals 'almost' exist in the sense that amplitudes stay small for a long time, but grow very slowly in a process called 'Arnold Diffusion'. This possibility is not excluded in the higher-dimensional case because an $n$-dimensional closed invariant surface in $2 n$-dimensional space does not completely enclose a $2 n$-dimensional volume unless $n=1$. (A circle will not hold water; this requires a closed sphere.) Whether this diffusion is a general property has not yet been proved. Arnold proved this for a particular system; however, it is generally believed that Arnold diffusion is generic. In spite of this the diffusion is quite slow.

(f) Ergodic Motion. In this case all of the energy surface gets covered; as the motion proceeds, the system gets to the neighborhood of every point on the energy surface. Statistical mechanics is based on the proposition that, with many degrees of freedom, this is the normal state of affairs. Then it can be shown that in infinite time the energy surface is covered uniformly in some sense.

Thus the characteristics of the motion of a Hamiltonian system are quite complex and not yet fully understood in the nonintegrable case. In this paper we will not attempt to cover all the types of motion discussed above. Instead we will discuss integrable systems and 'nearly integrable' systems. In the nearly integrable cases it is possible to use perturbative techniques quite successfully to study the motion in spite of the fact that these methods generally yield divergent series. However, it is now known that there are regions of phase space where the perturbation series do converge (the KAM theorem). We will use them here somewhat carefully and attempt to restrict their validity in a heuristic fashion. In addition the emphasis here will be on one-dimensional problems with time dependent Hamiltonians; however, many results will be stated in a general form.

We begin with a review of Hamiltonian dynamics and then discuss canonical transformations in some detail. We end with two detailed examples in which we study the behavior of nonlinear systems in phase space. The emphasis here is not to obtain the detailed motion of a point in phase space, but rather to illustrate the shapes of the curves in phase space to which the motion is confined. 
In this way one can determine stability without knowing the details of the initial conditions.

\section{HAMILTONIAN DYNAMICS}

\subsection{EQUATIONS OF MOTION}

The dynamical systems of interest here can be described by a Hamiltonian $H(q, p, t) . q$ is the coordinate, $p$ is the canonical momentum, and $t$ is the independent variable or time. In many cases the Hamiltonian is the sum of the kinetic energy $T$ and potential energy $V$ each written as a function of the coordinates and canonical momenta. The equations of motion can be derived from the Hamiltonian using Hamilton's equations:

$$
\frac{d q_{i}}{d t}=\frac{\partial H}{\partial p_{i}} \quad, \quad \frac{d p_{i}}{d t}=-\frac{\partial H}{\partial q_{i}}
$$

For example, consider a system of $n$ nonrelativistic particles interacting through a force law derivable from a potential. Then we have

$$
H=\frac{1}{2 m}\left(p_{1}^{2}+p_{2}^{2}+\cdots+p_{n}^{2}\right)+V\left(q_{1}, q_{2}, \cdots, q_{n}\right)
$$

and

$$
\frac{d q_{i}}{d t}=\frac{p_{i}}{m} \quad, \quad \frac{d p_{i}}{d t}=-\frac{\partial V}{\partial q_{i}}
$$

The above differential equations are simply Newton's Second Law for the $n$-particle system.

In the above example the canonical momenta were equal to the kinetic momenta. It is obvious that this is not true for more general Hamiltonians. Consider a nonrelativistic charged particle in an electromagnetic field with vector potential $\vec{A}(x, t)$ and scalar potential $\Phi(x, t)$. Then the Hamiltonian is given by

$$
H=\frac{1}{2 m}\left(\vec{p}-\frac{e}{c} \vec{A}(\bar{x}, t)\right)^{2}+e \Phi(\bar{x}, t)
$$

and the corresponding equations of motion are

$$
\begin{aligned}
v_{i} \equiv \frac{d x_{i}}{d t} & =\frac{p_{i}-\frac{e}{c} A_{i}}{m} \\
\frac{d p_{i}}{d t} & =-e \frac{\partial \Phi}{\partial x_{i}}-\frac{e}{c} \sum_{j} \frac{\left(p_{j}-\frac{e}{c} A_{j}\right)}{m} \frac{\partial A_{j}}{\partial x_{i}} .
\end{aligned}
$$


Note that in this case the canonical momenta and the kinetic momenta are related by

$$
m v_{i}=p_{i}-\frac{e}{c} A_{i}
$$

Using Eq. (2.6) to eliminate the canonical momenta in favor of the velocities, and recalling the relation of the electric and magnetic fields to the vector and scalar potentials, we find that Eq. (2.5) becomes

$$
\frac{d v}{d t}=\frac{e}{m}\left\{\vec{E}+\frac{\vec{v}}{c} \times \vec{B}\right\}
$$

Equation (2.7) is simply the Lorentz force equation for a nonrelativistic charged particle in an external electromagnetic field.

\subsection{SyMMETRY, INTEgRALS, AND CYCLIC COORDINATES}

If we examine Eq. (2.3), it is easy to see that if the Hamiltonian is independent of some coordinate $q_{m}$, then the corresponding canonical momentum $p_{m}$ is a constant of the motion. In this case $p_{m}$ is a first integral of the motion and the coordinate $q_{m}$ is called a 'cyclic' or 'ignorable' coordinate. In general, the existence of such an integral corresponds to a certain symmetry of the system. In this case the symmetry is the invariance of the equations of motion to translations in $q_{m}$. If $q_{m}$ is an angular coordinate, then the conjugate angular momentum is conserved, and the system is invariant with respect to a rotation in $q_{m}$.

In general for an $n$-dimensional system, Hamilton's equations constitute a system of $2 n$ ordinary first-order differential equations. In order to integrate such a system we need to know $2 n$ first integrals. In many cases, however, it is sufficient to know only $n$ independent integrals. In these cases each integral can be used to reduce the order of the system of equations by two rather than just one. These problems are called 'integrable', and the motion is confined to an $n$-dimensional surface in $2 n$-dimensional phase space.

In other cases $n$ independent integrals do not exist; these are called 'nonintegrable'. In these cases the trajectory can fill regions of phase space of dimension greater than $n$. The study of nonintegrable systems is still far from complete.

Although many of the differential equations which will be discussed here are, strictly speaking, nonintegrable, they are sufficiently close to integrable systems to admit approximate solutions. The precise nature of the breakdown of integrals when passing from an integrable system to a nonintegrable system is the subject of the KAM theorem mentioned previously. ${ }^{4}$ 


\subsection{MOTION NEAR A KNOWN PERIOdIC SOLUTION}

In many cases we are interested in the orbits of a system which are close to a known periodic solution. This periodic solution may or may not be easy to find; let us assume that we know it. Consider the Hamiltonian in Eq. (2.2) in two dimensions. This yields the equations of motion,

$$
\begin{aligned}
& m \bar{x}=-\frac{\partial V}{\partial x} \\
& m \bar{y}=-\frac{\partial V}{\partial y} .
\end{aligned}
$$

A periodic orbit $x_{0}(t)$ and $y_{0}(t)$ with period $T$ is defined to be one which closes on itself in time $T$. Thus it is defined by

$$
\begin{array}{ll}
m \ddot{x}_{0}=-\frac{\partial V}{\partial x}\left(x_{0}, y_{0}\right) \quad, & x_{0}(t+T)=x_{0}(t) \\
m \ddot{y}_{0}=-\frac{\partial V}{\partial x}\left(x_{0}, y_{0}\right), & y_{0}(t+T)=y_{0}(t) .
\end{array}
$$

Now cōnsider an orbit close to the periodic orbit and let

$$
\begin{aligned}
& \xi=x-x_{0} \\
& \eta=y-y_{0} .
\end{aligned}
$$

Then if we substitute into Eq. (2.8) and expand for small $\xi$ and $\eta$, we find

$$
\begin{aligned}
& m \ddot{\xi}=-\xi \frac{\partial^{2} V}{\partial x^{2}}\left(x_{0}, y_{0}\right)-\eta \frac{\partial^{2} V}{\partial x \partial y}\left(x_{0}, y_{0}\right) \\
& m \ddot{\eta}=-\xi \frac{\partial^{2} V}{\partial x \partial y}\left(x_{0}, y_{0}\right)-\eta \frac{\partial^{2} V}{\partial y^{2}}\left(x_{0}, y_{0}\right) .
\end{aligned}
$$

Thus, since $y_{0}$ and $x_{0}$ are periodic functions of $t$, we find a linear differential equation with periodic coefficients which can be derived from the Hamiltonian,

$$
H=\frac{p_{\xi}^{2}}{2 m}+\frac{p_{\eta}^{2}}{2 m}+\frac{1}{2}\left\{\frac{\partial^{2} V}{\partial x^{2}} \xi^{2}+2 \frac{\partial^{2} V}{\partial x \partial y} \xi \eta+\frac{\partial^{2} V}{\partial y^{2}} \eta\right\}
$$

where the derivatives of the potential are again evaluated at $\left(x_{0}, y_{0}\right)$. Note that the coefficients in the new Hamiltonian now depend periodically on time rather than being constant. Analysis of even the linearized problem is more difficult. In addition, the fact that the old Hamiltonian is a constant of the motion is now hidden. 
The stability or instability of the periodic orbit in question is determined by the solutions of Eq. (2.11). Thus the solutions of linear equations with periodic coefficients are evidently of fundamental importance. The solutions to this type of equation (Hill's equation) in one dimension will be discussed in Sections 2.6 and 4.2.

\subsection{Change of INDEPENDENT VARIABle ${ }^{2}$}

In many problems we are more interested in the orbits a particle describes in space than in the detailed time dependence of the solution. In this case we ask: how does $y$ behave as a function of $x$ ? Hamilton's equations in two dimensions are:

$$
\begin{array}{ll}
\frac{d x}{d t}=\frac{\partial H}{\partial p_{x}} \quad, \quad \frac{d y}{d t}=\frac{\partial H}{\partial p_{y}} \\
\frac{d p_{x}}{d t}=-\frac{\partial H}{\partial x} \quad, \quad \frac{d p_{y}}{d t}=-\frac{\partial H}{\partial y} .
\end{array}
$$

In addition we know that

$$
\begin{aligned}
& \frac{d y}{d x}=\frac{d y / d t}{d x / d t}=\frac{\partial H / \partial p_{y}}{\partial H / \partial p_{x}} \quad, \quad \frac{d p_{y}}{d x}=-\frac{\partial H / \partial y}{\partial H / \partial p_{x}} \\
& \frac{d t}{d x}=\frac{1}{\partial H / \partial p_{x}} \quad, \quad \frac{d H}{d x}=\frac{d H / d t}{d x / d t}=\frac{\partial H / \partial t}{\partial H / \partial p_{x}}
\end{aligned}
$$

From Eq. (2.14) it is clearly necessary to restrict

$$
\frac{d x}{d t}=\frac{\partial H}{\partial p_{x}} \neq 0
$$

That is, $x$ must increase or decrease monotonically with $t$.

Now instead of viewing the Hamiltonian as a function of the coordinates and momenta one can view $p_{x}$ as a function of $x, y, p_{y}, H$, and $t$. By comparing the total differentials of $p_{x}$ and $H$ one finds

$$
\begin{array}{ll}
\frac{\partial p_{x}}{\partial y}=-\frac{\partial H / \partial y}{\partial H / \partial p_{y}} \quad, \quad \frac{\partial p_{x}}{\partial p_{y}}=-\frac{\partial H / \partial p_{y}}{\partial H / \partial p_{x}} \\
\frac{\partial p_{x}}{\partial H}=\frac{1}{\partial H / \partial p_{x}} \quad, \quad \frac{\partial p_{x}}{\partial t}=\frac{-\partial H / \partial t}{\partial H / \partial p_{x}}
\end{array} .
$$

Thus, Hamilton's equations can be rewritten

$$
\frac{d y}{d x}=-\frac{\partial p_{x}}{\partial p_{y}} \quad, \quad \frac{d p_{y}}{d x}=\frac{\partial p_{x}}{\partial y} .
$$


and in addition

$$
\frac{d t}{d x}=\frac{\partial p_{x}}{\partial H} \quad, \quad \frac{d H}{d x}=\frac{-\partial p_{x}}{\partial t}
$$

Therefore $y$ and $p_{y}$ behave as if their motion is governed by a new Hamiltonian:

$$
\mathcal{K}=-p_{x}\left(y, p_{y}, t,-H, x\right)
$$

where $x$ now plays the role of the new 'time' variable. The new set of Hamilton's equations in terms of the new Hamiltonian are

$$
\begin{aligned}
& \frac{d y}{d x}=\frac{\partial \mathcal{H}}{\partial p_{y}} \quad, \quad \frac{\partial p_{y}}{\partial x}=-\frac{\partial \mathcal{H}}{\partial y} \\
& \frac{d t}{d x}=\frac{\partial \mathcal{H}}{\partial(-H)} \quad, \quad \frac{d(-H)}{d x}=-\frac{\partial \mathcal{H}}{\partial t} .
\end{aligned}
$$

Note that the equations have been supplemented with those for $H$ and $t .(-H)$ now plays the role of the momentum conjugate to $t$. For a 'conservative' system the Hamiltonian is independent of $t$ and thus energy is conserved. Therefore, in this case $t$ is an ignorable coordinate. Thus for a conservative system with $n$ degrees of freedom, we obtain a new system with $n-1$ degrees of freedom with a 'time' dependent Hamiltonian. Now the independent variable is one of the old coordinates, and the new Hamiltonian is the momentum conjugate to that coordinate expressed as a function of all other variables, including $H$ as a parameter. The actual time dependence can be obtained separately after solving the problem. This technique is particularly useful if the new time is an angle variable.

\subsection{The Motion of A Particle in aN ACCelerator}

A relativistic charged particle moving in a magnetic field can be described by the Hamiltonian

$H=c\left[m^{2} c^{2}+(\vec{p}-e \vec{A} / c)^{2}\right]^{1 / 2}$

where $A$ is the vector potential. It is useful to use a coordinate system based on a closed planar reference curve shown in Fig. 2.1. The coordinate system $(x, s, y)$ is similar to a cylindrical system, however, the radius of curvature may vary along the curve.

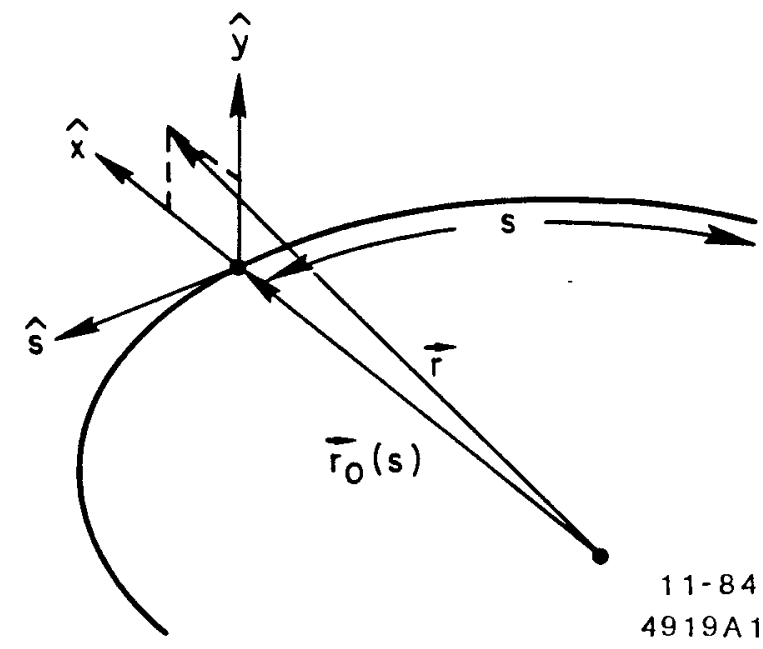

Fig. 2.1 The Coordinate System. 
If $\vec{r}$ is the coordinate of a particle in space and $\vec{r}_{0}$ is the point on the reference curve closest to $\vec{r}$, then

$$
s=\text { distance along the curve to the point } \vec{r}_{0}
$$

from a fixed origin somewhere on the curve;

$$
\begin{aligned}
& x=\text { horizontal projection of the vector } \vec{r}-\vec{r}_{0} ; \\
& y=\text { vertical projection of the vector } \vec{r}-\vec{r}_{0} ; \\
& \rho=\text { local radius of curvature. }
\end{aligned}
$$

The Hamiltonian written in terms of these coordinates is

$$
H=c\left[m^{2} c^{2}+\frac{\left(p_{s}-\frac{e}{c} A_{s}\right)^{2}}{\left(1+\frac{x}{\rho}\right)^{2}}+\left(p_{x}-\frac{e}{c} A_{x}\right)^{2}+\left(p_{y}-\frac{e}{c} A_{y}\right)^{2}\right]^{1 / 2}
$$

where $p_{x}$ and $p_{y}$ are projections of $\vec{p}$ onto the $x$ and $y$ direction and

$$
p_{s}=(\vec{p} \cdot \hat{s})\left(1+\frac{x}{\rho}\right) \text {. }
$$

Similar relations hold for $A_{s}, A_{x}$, and $A_{y}$. Note that $A_{s}$ as defined here is not simply the component of $\vec{A}$ in the $\hat{s}$ direction.

Further assume that the magnetic field in the neighborhood of the design orbit is given by

$$
\begin{aligned}
& B_{y}=-B_{0}(s)+B_{1}(s) x+\ldots \\
& B_{x}=B_{1}(s) y+\ldots
\end{aligned}
$$

and define the focusing function

$$
K_{1}(s)=\frac{B_{1}(s)}{B_{0}(s) \rho(s)} .
$$

Then the vector potential as defined above is given by

$$
A_{s}=-B_{0} \rho\left[\frac{x}{\rho}+\left(\frac{1}{\rho^{2}}-K_{1}\right) \frac{x^{2}}{2}+\frac{K_{1} y^{2}}{2}\right]+\ldots .
$$

Instead of working with the Hamiltonian in Eq. (2.21), we will use the results of Section 2.4 and choose $s$ as the new independent variable. Then the new Hamiltonian is

$$
K=\left(-p_{s}\right)=\frac{-e A_{s}}{c}-\left(1+\frac{x}{\rho}\right)\left[\frac{H^{2}}{c^{2}}-m^{2} c^{2}-p_{x}^{2}-p_{y}^{2}\right]^{1 / 2} .
$$


Since there is no time dependence, $H$ is a constant of the motion which we call $E$ ( the energy). If we expand for small deviations about the reference momentum, we find

$$
\nVdash=\left(p_{0}-p\right) \frac{x}{\rho}+p_{0}\left[\left(\frac{1}{\rho^{2}}-K_{1}\right) \frac{x^{2}}{2}+K_{1} \frac{y^{2}}{2}\right]+\frac{p_{x}^{2}}{2 p_{0}}+\frac{p_{y}^{2}}{2 p_{0}}
$$

where $p_{0}$ is defined to be the momentum on the reference orbit,

$$
p_{0}=\frac{e B_{0}}{c} \rho,
$$

and $p$ is the particle momentum,

$$
p^{2}=\frac{E^{2}}{c^{2}}-m^{2} c^{2}
$$

Considering a particle with the reference momentum $p_{0}$, the equations of motion are

$$
\begin{array}{ll}
\frac{d x}{d s}=\frac{p_{x}}{p_{0}}, & \frac{d y}{d s}=\frac{p_{x}}{p_{0}} \\
p_{x}^{\prime}=p_{0}\left(\frac{1}{\rho^{2}}-K_{1}\right) x & p_{y}^{\prime}=-p_{0} K_{1} y .
\end{array}
$$

In terms of $x$ and $y$ Eqs. (2.32) becomes

$$
\begin{aligned}
& x^{\prime \prime}=-\left(\frac{1}{\rho^{2}}-K_{1}\right) x \\
& y^{\prime \prime}=-K_{1} y .
\end{aligned}
$$

Equations (2.33) yield the motion of particles near the equilibrium orbit (usually called betatron oscillations). Because $K_{1}$ is dependent on $s$, the equations are not second-order equations with constant coefficients. The coefficients are periodic functions of $s$ since the equilibrium orbit is a closed curve. As we indicated in Section 2.3 these types of equations are called Hill's equations. There are some very general properties of the solutions of Hill's equation which will be discussed in the next section. 


\subsection{FLOQUET'S THEOREM ${ }^{9}$}

If $C$ is the length of the equilibrium orbit, then the coefficients of the linear equations (2.33) have period $C$ in $s$. Floquet's theorem gives the form of the solution of linear equations with periodic coefficients. Let us consider the general. second order equation

$$
x^{\prime \prime}+K(s) x=0
$$

where

$$
K(s+C)=K(s) .
$$

Suppose $x_{1}(s)$ and $x_{2}(s)$ are two linearly independent solutions of Eq. (2.34). Then the general solution $x(s)$ can be written in terms of $x_{1}$ and $x_{2}$ and two arbitrary constants $b_{1}$ and $b_{2}$,

$$
x(s)=b_{1} x_{1}(s)+b_{2} x_{2}(s) .
$$

noindent Because the differential equation has periodic coefficients, $x_{1}(s+C)$ and $x_{2}(s+C)$ satisfy the same equation, i.e.,

$$
x_{i}^{\prime \prime}(s+C)+K(s) x_{i}(s+C)=0 .
$$

Therefore, they can be expressed in terms of $x_{1}(s)$ and $x_{2}(s)$,

$$
\begin{aligned}
& x_{1}(s+C)=a_{11} x_{1}(s)+a_{12} x_{2}(s) \\
& x_{2}(s+C)=a_{21} x_{1}(s)+a_{22} x_{2}(s) .
\end{aligned}
$$

Combining Eq. (2.36) and (2.38), we have

$$
x(s+C)=\left(b_{1} a_{11}+b_{2} a_{21}\right) x_{1}(s)+\left(b_{1} a_{12}+b_{2} a_{22}\right) x_{2}(s) .
$$

We are looking for a solution called the 'normal' solution which satisfies the requirement

$$
x(s+C)=\lambda x(s)=\lambda\left(b_{1} x_{1}(s)+b_{2} x_{2}(s)\right) .
$$

If this is to be true for all $s$ then

$$
\left(\begin{array}{ll}
a_{11} & a_{12} \\
a_{21} & a_{22}
\end{array}\right)\left(\begin{array}{l}
b_{1} \\
b_{2}
\end{array}\right)=\lambda\left(\begin{array}{l}
b_{1} \\
b_{2}
\end{array}\right) .
$$


In order for Eq. (2.41) to have a nontrivial solution, the determinant of the coefficients must vanish; i.e.,

$$
\left|\begin{array}{cc}
a_{11}-\lambda & a_{12} \\
a_{21} & a_{22}-\lambda
\end{array}\right|=0
$$

or

$$
\lambda^{2}-\left(a_{11}+a_{22}\right) \lambda+\left(a_{11} a_{22}-a_{12} a_{21}\right)=0 .
$$

The constant term in Eq. (2.43) is the determinant of the matrix in Eq. (2.37). Since the differential equation we are studying is derivable from a Hamiltonian, the determinant is unity. Therefore the solutions of Eq. (2.42) are

where

$$
\lambda=e^{ \pm i \mu}
$$

$$
\cos \mu=\frac{a_{11}+a_{22}}{2}
$$

Note that $\mu$ can be complex. Since the determinant of the matrix $A$ is unity, the eigenvalues must be reciprocals; that is, if $\lambda$ is an eigenvalue, then $1 / \lambda$ is also an eigenvalue. This property generalizes to higher dimensions as well. In this case the eigenvalues come in reciprocal pairs.

We are now in a position to determine stability. To do this consider iterating the map of the normal solutions. This yields

$$
x(s+n C)=\lambda^{n} x(s) .
$$

If $x(s+n C)$ is to remain bounded in the limit as $n \rightarrow \infty$, then

$$
\|\lambda\| \leq 1
$$

However, for stability both normal solutions must be stable, i.e.,

$$
\left\|\frac{1}{\lambda}\right\| \leq 1
$$

To satisfy both Eq. (2.47) and Eq. (2.48) the magnitude of the eigenvalues of the matrix in Eq. (2.31) must be unity, and therefore $\mu$ must be a real quantity. From Eq. (2.45) this implies

or

$$
\left|a_{11}+a_{22}\right|<2
$$

$$
|\operatorname{Tr}(A)|<2
$$

where $\operatorname{Tr}$ stands for the trace. 
Finally it is useful to find the form of the normal solutions. We have from Eqs. (2.40) and (2.44)

$$
x(s+C)=e^{i \mu} x(s)
$$

Now we let

$$
x(s)=P(s) e^{i \mu s / C}
$$

and substitute into Eq. (2.51), which yields

$$
P(s+C)=P(s) .
$$

Therefore $P(s)$ is a periodic function with period $C$. Thus the two normal solutions to Eq. (2.34) are the product of an exponential function of $s$ and a periodic function of $s$, the distance along the reference orbit.

\subsection{HIGHER-ORDER TERMS}

To obtain Eq. (2.33) in Section 2.5 it was necessary to linearize the equations of motion or to expand the Hamiltonian to quadratic terms. In doing so there were higher-order terms which were neglected. These are basically of two types: 1) Higher-order 'geometric' terms are those which come directly in the magnetic field or vector potential. These are due to deliberate or inadvertent nonlinearites in the magnetic field. 2) Higher-order 'kinematic' terms come from the expansion of the square root in Eq. (2.28).

Since these higher-order terms are present in an actual accelerator, we must ask if they alter the linear stability calculated in Section 2.6. One might think that if the coefficients of the nonlinear terms are sufficiently small then the motion is essentially linear and stability is assured. This is in fact true for some cases.

On the other hand this is not true in general. To see this in a simple example consider the Hamiltonian

$$
H(x, p, \theta)=\frac{1}{2}\left(p^{2}+\frac{1}{9} x^{2}\right)+\frac{\epsilon}{3} x^{3} \cos \theta
$$

where $\theta$ is the independent variable. The differential equation is then

$$
\frac{d^{2} x}{d \theta^{2}}=-\frac{x}{9}-\epsilon x^{2} \cos \theta
$$

To see if the solution is stable, let us consider a solution which deviates by a small amount from the linear solution:

$$
x=a \cos (\theta / 3)+y
$$


where $y$ is a small quantity (of order $\epsilon$ ). Now if we substitute into Eq. (2.55) and expand for small $y$, we find

$$
\frac{d^{2} y}{d \theta^{2}}+\frac{1}{9} y=-\frac{\epsilon a^{2}}{2}\left[\cos \theta+\frac{1}{2} \cos \left(\frac{\theta}{3}\right)+\frac{1}{2} \cos \left(\frac{5 \theta}{3}\right)\right] \text {. }
$$

The equation for $y$ is then just a driven harmonic oscillator; however, one of the driving terms has the same frequency as the frequency of free oscillations. In this case the amplitude of the solution grows linearly in $\theta$. Thus, this equation indicates an instability. This is an example of a nonlinear resonance. The resonance occurred because the linear oscillation frequency was $1 / 3$; however, there is still another possibility. Perhaps the small terms in $y$ which were neglected in Eq. (2.57) will shift the frequency of the oscillator off resonance. For the particular example treated here, this does not happen. In other cases discussed in Sections 4 and 5 the nonlinear shifts in frequency do stabilize the motion. This will be discussed in more detail after we have developed more tools to understand nonlinear behavior.

\section{CANONICAL TRANSFORMATIONS}

A dynamical system is described in terms of a certain set of variables, coor-dinates and canonically conjugate momenta. Sometimes it is more convenient to express the equations of motion in terms of different variables which are functions of the old ones. It is desirable to have the new coordinates again in Hamiltonian form; that is, if $Q$ and $P$ are the new coordinates, then

$$
\frac{d Q}{d t}=\frac{\partial K(Q, P, t)}{\partial P} \quad, \quad \frac{d P}{d t}=-\frac{\partial K(Q, P, t)}{\partial Q}
$$

where $K(Q, P, t)$ is the new Hamiltonian. The question is then to find those transformations which accomplish this.

\subsection{The Generating Function of a Canonical Transformation ${ }^{1}$}

Hamilton's equations of motion can be derived from a variational principle. For a system described by a Hamiltonian $H(q, p, t)$, the Lagrangian function is

$$
\mathcal{L}(q, \dot{q}, t)=\sum_{i} p_{i} \dot{q}_{i}-H\left(q_{i}, p_{i}, t\right)
$$

Consider the evolution of the system from $t_{1}$ to $t_{2}$ and the action integral

$$
S=\int_{t_{1}}^{t_{2}} \mathcal{L}(q(t), \dot{q}(t), t) d t
$$


Then we vary the function $q(t)$ so that the end points are fixed, and ask for what $q(t)$ is the action integral stationary. The answer can be found from the calculus of variations. $q(t)$ must satisfy

$$
\frac{d}{d t} \frac{\partial \mathcal{L}}{\partial \dot{q}}-\frac{\partial \mathcal{L}}{\partial q}=0
$$

which is equivalent to

$$
\frac{d\left(p_{i}\right)}{d t}+\frac{\partial H}{\partial q_{i}}=0 \quad, \quad \dot{q}_{i}=\frac{\partial H}{\partial p_{i}} .
$$

Equations (3.5) are Hamilton's equations of motion.

Now, with new variables $Q$ and $P$ and a new Hamiltonian $K$, Hamilton's principle must again be valid

$$
\delta S^{\prime}=\delta \int_{t_{1}}^{t_{2}}\left[\sum_{i} P_{i} \dot{Q}_{i}-K(Q, P, t)\right] d t=0 .
$$

Therefore either $S=S^{\prime}$, or they differ at most by a total time derivative of some function $W$.

This function must be a function of the new and old variables. However, only $2 n$ of these are independent for an $n$-dimensional problem since there are $2 n$ transformation equations relating the new and old coordinates and momenta. Consider a function which depends only on the new and old coordinates. That is

$$
W=F_{1}(q, Q, t) \text {. }
$$

Then we must have

$$
\sum_{i} p_{i} \dot{q}_{i}-H=\sum_{i} P_{i} \dot{Q}_{i}-K+\frac{d F_{1}}{d t}
$$

Now if we expand the total time derivative we have

$$
\sum_{i} \dot{q}_{i}\left(p_{i}-\frac{\partial F_{1}}{\partial q_{i}}\right)-\sum_{i} \dot{Q}_{i}\left(P_{i}+\frac{\partial F_{1}}{\partial Q_{i}}\right)-\left(H-K+\frac{\partial F_{1}}{\partial t}\right)=0 .
$$

For Eq. (3.9) to hold identically, the coefficients of $\dot{q}$ and $\dot{Q}$ must vanish because $q$ and $Q$ are the $2 n$ independent variables. Thus we must have

$$
\begin{aligned}
& p_{i}=\frac{\partial F_{1}}{\partial q_{i}} \quad, \quad P_{i}=-\frac{\partial F_{1}}{\partial Q_{i}} \\
& K=H+\frac{\partial F_{1}}{\partial t} .
\end{aligned}
$$

Equations (3.10) specify the relations between the old and new variables in a canonical transformation. The first two of these equations can be solved for $q$ 
and $p$ in terms of $Q$ and $P$. The new Hamiltonian is then given by the third equation in (3.10);

$$
K(Q, P, t)=H(q(Q, P, t), p(Q, P, t), t)+\frac{\partial F_{1}}{\partial t}(q(Q, P, t), Q, t) .
$$

$F_{1}(q, Q, t)$ is called the generating function of the canonical transformation in Eqs. (3.10). Rather than choosing the old coordinates and new coordinates $(q, Q)$ as variables, we could have chosen the old coordinates and new momenta $(q, P)$. In this case we have a different generating function $F_{2}(q, P, t)$, and a different set of equations for the canonical transformation

$$
\begin{aligned}
p & =\frac{\partial F_{2}}{\partial q}(q, P, t), \\
Q & =\frac{\partial F_{2}}{\partial P}(q, P, t), \\
K & =H+\frac{\partial F_{2}}{\partial t}(q, P, t) .
\end{aligned}
$$

$F_{2}$ and $F_{1}$ are related by a Legendre transformation.

The equations of a canonical transformation can be viewed in many different ways. We could start with the relationship between the coordinates, derive the generating function which yields that, and then find the new momenta and new Hamiltonian. Alternatively we could begin with a new Hamiltonian, solve for the generating function and then calculate the new coordinates. In the next sections we show some examples.

\subsection{ACTION-ANGLE VARIABLES FOR THE HARMONIC OSCILLATOR}

In this section we consider a problem that we know how to solve. The harmonic oscillator Hamiltonian is

$$
H=\frac{p^{2}}{2}+\frac{\omega^{2} x^{2}}{2},
$$

and the solution of the equation of motion is

$$
\begin{aligned}
& x=a \cos \left(\omega t+\phi_{0}\right) \\
& p=-a \omega \sin \left(\omega t+\phi_{0}\right),
\end{aligned}
$$

where $a$ and $\phi_{0}$ are two arbitrary constants. The motion is confined to an ellipse in phase space. Note that the Hamiltonian is independent of the time and is thus a constant of the motion. Therefore the constant $a$ is related to the constant value of $H$. 
Now we would like to change to a set of variables for which the new Hamiltonian is a function only of the new momentum. Since we already know the solution above, we can use it to construct these new coordinates. Eq. (3.14) suggests we consider a transformation of the form

$$
\begin{aligned}
& x=a(J) \cos (\phi) \\
& p=-a(J) \omega \sin (\phi)
\end{aligned}
$$

where $J$ and $\phi$ are the new momentum and coordinate respectively. $a(J)$ is some as yet unspecified function of the new momentum. To accomplish the transformation we will use a generating function of the first type discussed in the previous section. From the transformation equations in Eq. (3.10), we need to find the old momentum $p$ in terms of the new and old coordinates. This can be done by combining the two equations in Eq. (3.15) to yield

$$
p=-\omega x \tan \phi .
$$

The equation for the generating function can be integrated to yield

$$
F_{1}(x, \phi)=-\frac{\omega x^{2}}{2} \tan \phi .
$$

Solving for the new momentum we find

$$
J=\frac{\left(\omega^{2} x^{2}+p^{2}\right)}{2 \omega},
$$

and the complete set of transformation equations now reads

$$
\begin{aligned}
x & =\sqrt{2 J / \omega} \cos \phi \\
p & =-\sqrt{2 J \omega} \sin \phi \\
K & =\frac{p^{2}}{2}+\frac{\omega^{2} x^{2}}{2}=\omega J .
\end{aligned}
$$

The new momentum $J$ is called the action variable while the new coordinate $\phi$ is the angle variable. It is not hard to see that if the Hamiltonian has the units of energy, $J$ has the units of an action.

These coordinates are very useful for studying problems which differ from a harmonic oscillator only by the addition of small nonlinear terms. 


\subsection{DEVIATION FROM A KNOWN SOLUTION}

In Section 2.5 we saw that deviations from a known periodic solution to a differential equation obeyed a linear differential equation with periodic coefficients. It is useful to derive a somewhat more general result using canonical transformations. Consider a Hamiltonian $H$ with a known particular solution $q_{0}(t)$ and $p_{0}(t)$. For cases of interest this is the periodic solution to an inhomogeneous differential equation. This known solution satisfies

$$
\begin{aligned}
& \frac{d q_{0}(t)}{d t}=\frac{\partial H}{\partial p}\left(q_{0}(t), p_{0}(t), t\right) \\
& \frac{d p_{0}(t)}{d t}=-\frac{\partial H}{\partial q}\left(q_{0}(t), p_{0}(t), t\right) .
\end{aligned}
$$

We would like to perform a canonical transformation to new coordinates and momenta which are close to the particular solution. Let the new coordinates and momenta be given by

$$
\begin{aligned}
& Q=q-q_{0}(t) \\
& P=p-p_{0}(t) .
\end{aligned}
$$

Now if we use a generating function of the second type the equations of the transformation are given by

$$
\begin{gathered}
p=\frac{\partial F_{2}}{\partial q}=P+p_{0}(t) \\
Q=\frac{\partial F_{2}}{\partial P}=q-q_{0}(t)
\end{gathered}
$$

which can be integrated to yield the generating function

$$
F_{2}(q, P, t)=\left[q-q_{0}(t)\right]\left[P+p_{0}(t)\right] .
$$

Then if we use Eq. (3.12) for the new Hamiltonian and expand for small $Q$ and $P$, we find

$$
\begin{aligned}
K & =H\left(q_{0}(t), p_{0}(t), t\right)+\dot{p}_{0}(t) q_{0}(t)+\frac{1}{2}\left[\left(H_{q q}\left(q_{0}(t), p_{0}(t), t\right)\right] Q^{2}\right. \\
& +\frac{1}{2} H_{p p}\left(q_{0}(t), p_{0}(t), t\right) P^{2}+H_{p q}\left(q_{0}(t), p_{0}(t), t\right) Q P
\end{aligned}
$$

where the subscripts denote partial differentiation. Thus the Hamiltonian consists of two types of terms, those which depend only on the time and those which 
are quadratic and higher-order functions of $Q$ and $P$ with time-dependent coefficients. The terms in the Hamiltonian which are not functions of $Q$ and $P$ do not affect the differential equation for $Q$ and $P$ and thus can be ignored. If the known solution is a periodic one, the lowest-order terms which contribute to the differential equations are second-order with periodic coefficients. Thus the differential equations are linear with periodic coefficients.

Particular solutions which are periodic are fixed points of the one-period mapping generated by the differential equation. The transformation above has moved that fixed point to the origin in the new coordinate system. This is easily seen if we write the condition for a fixed point $\left(Q_{0}, P_{0}\right)$ :

$$
\begin{aligned}
& \partial H / \partial Q=0 \\
& \partial H / \partial P=0
\end{aligned} .
$$

From Eq. (3.25) this is satisfied for

$$
Q_{0}=0, \quad P_{0}=0
$$

There may also be other fixed points of this system or other periodic orbits in the new variables. These periodic orbits are fixed points of mappings through different periods and thus the above process can be performed again.

Not surprisingly we will once again find quadratic Hamiltonians with periodic coefficients; that is, linear differential equations with periodic coefficients. Since these types of equations are so ubiquitous, we return to them in the next chapter.

\section{LINEAR EQUATIONS WITH PERIODIC COEFFICIENTS ${ }^{10}$}

There have been many useful techniques developed for linear equations with periodic coefficients in the context of alternating gradient focusing for particle accelerators or storage rings. ${ }^{10}$ In this section we follow Ref. 10 to develop these, now standard, techniques in one dimension. The matrix approach is used initially to understand stability and introduce the very important function $\beta$, the Courant-Snyder amplitude function. Finally we show a canonical transformation which changes the Hamiltonian to that for a harmonic oscillator. The subject of this section as in Section 2.6 is the solution of the differential equation

$$
\frac{d^{2} y}{d s^{2}}+K(s) y=0
$$


which can be derived from the Hamiltonian

$$
H=\frac{p^{2}}{2}+\frac{K(s) y^{2}}{2}
$$

$y$ represents either horizontal or vertical displacement, and $K$ satisfies the periodicity relation

$$
K(s+C)=K(s) .
$$

Here $C$ is the circumference of the equilibrium orbit.

In the alternating gradient synchrotron or storage ring the magnetic "lattice" ideally consists of $N$ identical sections or "unit cells", so that $K$ also satisfies the stronger periodicity relation

$$
K(s+L)=K(s) \quad ; \quad L=C / N
$$

\subsection{THE MATRIX APPROACH}

The solution of any linear second order differential equation of the form (4.2), whether or not $K$ is periodic, is uniquely determined by the initial values of $y$ and its derivative $y^{\prime}$ :

$$
\begin{aligned}
& y(s)=a y\left(s_{0}\right)+b y^{\prime}\left(s_{0}\right) \\
& y^{\prime}(s)=c y\left(s_{0}\right)+d y^{\prime}\left(s_{0}\right)
\end{aligned}
$$

or, in matrix notation,

$$
Y(s)=\left[\begin{array}{c}
y(s) \\
y^{\prime}(s)
\end{array}\right]=M\left(s \mid s_{0}\right) Y\left(s_{0}\right)=\left[\begin{array}{ll}
a & b \\
c & d
\end{array}\right]\left[\begin{array}{c}
y\left(s_{0}\right) \\
y^{\prime}\left(s_{0}\right)
\end{array}\right] .
$$

The usefulness of the matrix formulation (4.6) arises mainly from two features: In the first place, this formulation clearly separates the properties of the general solution of the problem from the features characterizing any particular solution. That is, the matrix $M\left(s \mid s_{0}\right)$ depends only on the function $K(s)$ between $s_{0}$ and $s$, and not on the particular solution. Secondly, the matrix for any interval made up of sub-intervals is just the product of the matrices for the sub-intervals, that is,

$$
M\left(s_{2} \mid s_{0}\right)=M\left(s_{2} \mid s_{1}\right) M\left(s_{1} \mid s_{0}\right)
$$

as is easily verified. 
The determinant of the matrix $M$ is equal to unity, because Eq. (4.1) does not contain any first-derivative terms.

For the particular case of constant $K$ the matrix takes the form

$$
M\left(s \mid s_{0}\right)=\left[\begin{array}{cc}
\cos \phi & K^{-1 / 2} \sin \phi \\
-K^{1 / 2} \sin \phi & \cos \phi
\end{array}\right]
$$

where $\phi=K^{1 / 2}\left(s-s_{0}\right)$. If $K$ is negative, a more convenient way of writing this is

$$
M=\left[\begin{array}{cc}
\cosh \psi & (-K)^{-1 / 2} \sinh \psi \\
(-K)^{1 / 2} \sinh \psi & \cosh \psi
\end{array}\right],
$$

where $\psi=(-K)^{1 / 2}\left(s-s_{0}\right)$. For an interval of length $l$ in which $K=0$,

$$
M=\left[\begin{array}{ll}
1 & l \\
0 & 1
\end{array}\right] .
$$

For an interval in which $K$ is piecewise constant the matrix is the product of the appropriate matrices of forms (4.8) to (4.10).

In the periodic systems we are considering here the matrices of particular interest are those which characterize the motion of the particle through a whole period. We write

$$
M(s)=M(s+L \mid s) ;
$$

this is the matrix for passage through one period, starting from $s$. Its elements are periodic functions of $s$ with period $L$. The matrix for passage through one revolution is then

$$
M(s+N L \mid s)=[M(s)]^{N},
$$

and that for passage through $k$ revolutions is $[M(s)]^{N k}$.

In order for the motion to be stable as defined above, it is necessary and sufficient that all the elements of the matrix $M^{N k}$ remain bounded as $k$ increases indefinitely. To obtain the condition for this, we consider the 
eigenvalues of the matrix $M(s)$, that is, those numbers $\lambda$ for which the characteristic matrix equation

$$
M Y=\lambda Y
$$

possesses nonvanishing solutions. The eigenvalues are the solutions of the determinantal equation

$$
|M-\lambda I|=0
$$

or, more fully,

$$
\lambda^{2}-\lambda(a+d)+1=0,
$$

where we have made use of the fact that Det $M=a d-b c=1$. If we define

$$
\cos \mu=\frac{1}{2} \operatorname{Tr} M=\frac{1}{2}(a+d),
$$

the two solutions of (4.14) are

$$
\lambda=\cos \mu \pm i \sin \mu=e^{ \pm i \mu} .
$$

The quantity $\mu$ will be real if $|a+d| \leq 2$, and imaginary or complex if $|a+d|>2$.

Let us now assume that $|a+d| \neq 2$. Then the matrix $M$ may be written in a form which exhibits the eigenvalues and other properties explicitly. We define $\cos \mu$ by (4.15), and define $\alpha, \beta$, and $\gamma$ by

$$
\begin{aligned}
a-d & =2 \alpha(s) \sin \mu, \\
b & =\beta(s) \sin \mu, \\
c & =-\gamma(s) \sin \mu ;
\end{aligned}
$$

the condition Det $M=1$ becomes

$$
\beta \gamma-\alpha^{2}=1
$$

The matrix $M$ may now be written as

$$
M=\left[\begin{array}{cc}
\cos \mu+\alpha \sin \mu & \beta \sin \mu \\
-\gamma \sin \mu & \cos \mu-\alpha \sin \mu
\end{array}\right]=I \cos \mu+J \sin \mu
$$


where $I$ is the unit matrix, and

$$
J=\left[\begin{array}{cc}
\alpha & \beta \\
-\gamma & -\alpha
\end{array}\right]
$$

is a matrix with zero trace and unit determinant, satisfying

$$
J^{2}=-I
$$

It should be noted that the trace of $M$, and therefore $\mu$, is independent of the reference point $s$. For, by virtue of (4.7), we have for any $s_{1}$ and $s_{2}$

$$
M\left(s_{2}+L \mid s_{1}\right)=M\left(s_{2}\right) M\left(s_{2} \mid s_{1}\right)=M\left(s_{2} \mid s_{1}\right) M\left(s_{1}\right),
$$

so that

$$
M\left(s_{2}\right)=M\left(s_{2} \mid s_{1}\right) M\left(s_{1}\right)\left[M\left(s_{2} \mid s_{1}\right)\right]^{-1} .
$$

Thus $M\left(s_{1}\right)$ and $M\left(s_{2}\right)$ are related by a similarity transformation, and therefore have the same trace and the same eigenvalues. On the other hand, the matrix $M(s)$ as a whole does depend on the reference point $s$. Thus the elements $\alpha, \beta$, $\gamma$ of the matrix $J$ are functions of $s$, periodic with period $L$.

Because of Eq. (4.21), the combination $I \cos \mu+J \sin \mu$ has properties similar to those of the complex exponential $e^{i \mu}=\cos \mu+i \sin \mu$; in particular, it is easily seen that, for any $\mu_{1}$ and $\mu_{2}$

$$
\left(I \cos \mu_{1}+J \sin \mu_{1}\right)\left(I \cos \mu_{2}+J \sin \mu_{2}\right)=I \cos \left(\mu_{1}+\mu_{2}\right)+J \sin \left(\mu_{1}+\mu_{2}\right) .
$$

The $k^{\text {th }}$ power of the matrix $M$ is thus

$$
M^{k}=(I \cos \mu+J \sin \mu)^{k}=I \cos k \mu+J \sin k \mu,
$$

and the inverse is

$$
M^{-1}=I \cos \mu-J \sin \mu
$$

It follows from (4.25) that if $\mu$ is real the matrix elements of $M^{k}$ do not increase indefinitely with increasing $k$ but rather oscillate; on the other hand, if $\mu$ is not real, $\cos k \mu$ and $\sin k \mu$ increase exponentially, and therefore the matrix elements do the same. Therefore, the motion is stable if $\mu$ is real, i.e., if $|a+d|<2$, and unstable if $|a+d|>2$. 
Thus, to summarize, the matrix approach can be used explicitly to construct the periodic matrix elements $a, b, c$ and $d$. Once the one-turn matrix at a point $s_{0}$ is known, its trace can be calculated. This yields $\mu$, which can then be used to calculate $\alpha, \gamma$ and $\beta$ at the point $s_{0}$. The values of $\alpha, \gamma$ and $\beta$ at other points can then be calculated via the similarity transformation in Eq. (4.23). In this case the matrix elements change but $\mu$ remains fixed and thus the change is entirely due to $\alpha, \gamma$ and $\beta$.

These parameters play a major role in determining the details of the motion. In particular, $\beta$ determines the maximum local amplitude of transverse oscillations. This is demonstrated in the next section.

\subsection{The Phase-Amplitude Form of the Solution}

Let us return to the form of the solution given in Eq. (2.52) which we rewrite

$$
y_{1}(s)=w(s) e^{i \psi(s)},
$$

where, for the moment, we impose no particular conditions on the functions $w$ and $\psi$. It is easily verified by substitution into Eq. (4.1) that, if $w$ and $\psi$ satisfy

$$
w^{\prime \prime}+K w-\frac{1}{w^{3}}=0
$$

and

$$
\psi^{\prime}=\frac{1}{w^{2}},
$$

then $y_{1}$ as defined by Eq. (4.27) is indeed a solution. In addition

$$
y_{2}(s)=w(s) e^{-i \psi(s)}
$$

is also a solution and $y_{1}$ and $y_{2}$ are linearly independent. Therefore any solution of (4.1) is a linear combination of $y_{1}$ and $y_{2}$. We can therefore write the matrix $M\left(s_{2} \mid s_{1}\right)$ in terms of the solutions $y_{1}$ and $y_{2}$ or, what amounts to the same thing, in terms of the functions $w$ and $\psi$. We obtain

$$
\begin{aligned}
& M\left(s_{2} \mid s_{1}\right)= \\
& {\left[\begin{array}{cc}
\frac{w_{2}}{w_{1}} \cos \psi-w_{2} w_{1}^{\prime} \sin \psi & w_{1} w_{2} \sin \psi \\
\frac{-1-w_{1} w_{1}^{\prime} w_{2} w_{2}^{\prime}}{w_{1} w_{2}} \sin \psi-\left(\frac{w_{1}^{\prime}}{w_{2}}-\frac{w_{2}^{\prime}}{w_{1}}\right) \cos \psi & \frac{w_{1}}{w_{2}} \cos \psi+w_{1} w_{2}^{\prime} \sin \psi
\end{array}\right]}
\end{aligned}
$$

where $\psi$ stands for $\psi\left(s_{2}\right)-\psi\left(s_{1}\right), w_{1}$ for $w\left(s_{1}\right)$, etc. 
We now consider the case where $s_{2}-s_{1}$ is just one period of $K(s)$, i.e., $s_{2}-s_{1}=L$. The matrix $M$ is then identical with the matrix (4.19). If we now require that $w(s)$ be a periodic function of $s$, then $w_{1}=w_{2}$ and $w_{1}^{\prime}=w_{2}^{\prime}$, and the forms (4.31) and (4.19) are identical provided we make the identifications

$$
\begin{gathered}
\psi\left(s_{2}\right)-\psi\left(s_{1}\right)=\mu, \\
w^{2}=\beta, \\
w w^{\prime}=-\alpha,
\end{gathered}
$$

from which follows automatically

$$
\frac{1+\left(w w^{\prime}\right)^{2}}{w^{2}}=\frac{1+\alpha^{2}}{\beta}=\gamma .
$$

This identification is legitimate if we can show that $\beta^{1 / 2}-$ which is, of course, periodic-satisfies the differential Eq. (4.28) and that

$$
\beta^{\prime}=-2 \alpha
$$

Te prove this, consider the matrix for the transformation from $s+d s$ to $s+L+d s$. This matrix is, by (4.23),

$$
M(s+d s)=M(s+d s \mid s) M(s)[M(s+d s \mid s)]^{-1} .
$$

For infinitesimal $d s$,

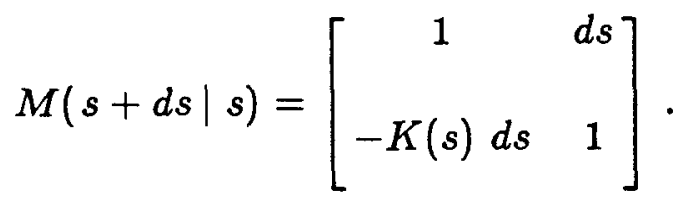

Substituting (4.38) and (4.19) into (4.37) we find

$$
M(s+d s)=M(s)+\left[\begin{array}{cc}
(K \beta-\gamma) \sin \mu & -2 \alpha \sin \mu \\
-2 K \alpha \sin \mu & -(K \beta-\gamma) \sin \mu
\end{array}\right] d s,
$$

so that (4.36) is indeed valid, and furthermore

$$
\alpha^{\prime}=-\frac{1}{2} \beta^{\prime \prime}=K \beta-\gamma=K \beta-\frac{1+\alpha^{2}}{\beta}
$$

and

$$
\gamma^{\prime}=2 K \alpha
$$


With the aid of (4.36) and (4.40) it is easily verified that $\beta^{1 / 2}$ does indeed satisfy (4.28), and is therefore a periodic solution of that equation. Now (4.33) and (4.34) are justified, while (4.32) becomes the very important relation

$$
\mu=\int_{0}^{L} \frac{d s}{\beta} .
$$

Equation (4.42) may be regarded as the definition of $\mu$. It is consistent with the previous definition, (4.15), but has the advantage of being unambiguous, while (4.15) only defines $\mu$ modulo $2 \pi$.

If we consider an accelerator of circumference $C=N L$ with $N$ identical unit cells, the phase change per revolution is, of course, $N \mu$. A useful number is

$$
\nu=\frac{N \mu}{2 \pi}=\frac{1}{2 \pi} \int_{s}^{s+C} \frac{d s^{\prime}}{\beta} ;
$$

this is the number of betatron oscillation wavelengths in one revolution. (In the European literature on accelerators this number is often denoted by $Q$.) A useful interpretation of $\nu$ is as the frequency of betatron oscillations measured in units of the frequency of revolution; we shall generally refer to $\nu$ simply as the frequency of oscillations or tune.

The two particular solutions $y_{1}$ and $y_{2}$ may now be written as

$$
y_{\frac{1}{2}}=\beta^{1 / 2}(s) e^{ \pm i \nu \phi(s)}
$$

where

$$
\phi(s)=\int \frac{d s}{\nu \beta}
$$

is a function which increases by $2 \pi$ every revolution, and whose derivative is periodic. The general solution of (4.1) is

$$
y(s)=a \beta^{1 / 2} \cos [\nu \phi(s)+\delta],
$$

where $a$ and $\delta$ are arbitrary constants. This is a pseudo-harmonic oscillation with varying amplitude $\beta^{1 / 2}(s)$ and varying instantaneous wavelength

$$
\lambda=2 \pi \beta(s) .
$$

Incidentally, the relation (4.47) between the amplitude and the wavelength is formally just the same as in the WKB solution of the problem of the harmonic 
oscillator with varying wavelength; however, the relation between the wavelength and the parameters of the differential equation is not as simple as in the WKB problem.

\subsection{ACtion-ANGLE VARIABLES}

Now let us assume that we have explicitly calculated $\beta(s)$ and $\phi(s)$. Then it is useful to construct action-angle variables for this problem in a way completely analogous to the harmonic oscillator in Section 3.2. To do this we first write the solution for both the position and momentum:

$$
\begin{aligned}
& y=a \beta^{1 / 2} \cos (\nu \phi(s)+\delta) \\
& p=-a \beta^{-1 / 2}\left[\sin (\nu \phi(s)+\delta)-\frac{\beta^{\prime}}{2} \cos (\nu \phi(s)+\delta)\right] .
\end{aligned}
$$

The momentum equation is obtained by simply differentiating the equation for $y$.

Now let us search for a canonical transformation of the form

$$
\begin{aligned}
& y=a(J) \beta^{1 / 2} \cos \psi \\
& p=-a(J) \beta^{-1 / 2}\left[\sin \psi-\frac{\beta^{\prime}}{2} \cos \psi\right]
\end{aligned}
$$

where $J$ and $\psi$ are the new momentum and coordinate respectively.

We will use a generating function of the first type; therefore, we need the old momenta $p$ in terms of the new and old coordinates. Combining the two equations in (4.49) yields

$$
p=-\frac{y}{\beta}\left(\tan \psi-\frac{\beta^{\prime}}{2}\right)
$$

Therefore, Eq. (3.10) for the generating function can be integrated to yield

$$
F_{1}(x, \psi)=-\frac{y^{2}}{2 \beta}\left[\tan \psi-\frac{\beta^{\prime}}{2}\right]
$$

Solving for the new momenta in terms of the old coordinates and momenta, we find

$$
J=\frac{1}{2 \beta}\left[y^{2}+\left(\beta y^{\prime}-\frac{\beta^{\prime} y}{2}\right)^{2}\right]
$$


and the complete set of transformation equations becomes

$$
\begin{aligned}
y & =\sqrt{2 J \beta} \cos \psi, \\
p & =-\sqrt{2 J / \beta}\left(\sin \psi-\frac{\beta^{\prime}}{2} \cos \psi\right), \\
H_{1} & =H+\partial F_{1} / \partial s=J / \beta(s) .
\end{aligned}
$$

The differential relations for $\beta$ in Eq. (4.40) have been used to simplify the new Hamiltonian.

In these new coordinates the solution of the equations of motion is

$$
\begin{aligned}
J & =\text { constant } \\
\psi(s) & =\psi(0)+\int_{0}^{s} \frac{d s^{\prime}}{\beta\left(s^{\prime}\right)} .
\end{aligned}
$$

Note that in the process we have explicitly constructed an invariant, $J$. Equation (4.52) for the invariant is the equation of an ellipse in phase space which rotates periodically in $s$. If a particle has initial conditions which begin on some ellipse given by $J_{0}$, then the coordinates and momentum of that particle always stay on that ellipse.

Looking at it in another way, consider a single particle traversing the periodic focusing structure and plot its position and momentum in phase space each time it passes $s=s_{0}$. Then, the locus of those points is an ellipse in phase space. At points other than $s_{0}$, the ellipse so generated evolves according to Eq. (4.52).

The invariant $J$ is simply related to the area enclosed by the ellipse:

$$
\text { Area enclosed }=2 \pi J \text {. }
$$

In accelerator and storage ring terminology there is a quantity called the emittance which is closely related to this invariant. The emittance, however, is a property of a distribution of particles, not a single particle. Consider a Gaussian distribution in amplitudes. Then the (rms) emittance, $\epsilon$, is given by

$$
\left(y_{\mathrm{rms}}\right)^{2}=\beta(s) \epsilon .
$$

In terms of the action variable, $J$, this can be rewritten

$$
\epsilon=\langle J\rangle
$$

where the bracket indicates an average over the distribution in $J$. 
Finally note that the form of the new Hamiltonian is not precisely that of a harmonic oscillator in that the phase does not advance uniformly. This of course causes no difficulty in that both cases are trivial to solve. However, it is possible to perform another canonical transformation to coordinates which have a uniformly advancing phase. This is accomplished with the canonical transformation:

$$
\begin{aligned}
F_{2}\left(\psi, J_{1}, s\right) & =J_{1}\left[\frac{2 \pi \nu s}{C}-\int_{0}^{s} \frac{d s^{\prime}}{\beta}\right]+\psi J_{1}, \\
\psi_{1} & =\psi+\frac{2 \pi \nu s}{C}-\int_{0}^{s} \frac{d s^{\prime}}{\beta}, \\
J_{1} & =J \\
H_{1} & =\frac{2 \pi \nu}{C} J_{1} \equiv \frac{\nu}{R} J_{1} .
\end{aligned}
$$

In these new coordinates the oscillating part of the phase advance has been extracted leaving only the average phase advance. Either these coordinates or the previous set can be used in the next chapter on canonical perturbation theory. We will use the second set in the next section since no reference is made to a specific problem. In the later sections we will use the first set $(J, \psi)$ since this simplifies the notation in spite of the fact that one must integrate to obtain the phase advance.

\section{CANONICAL PERTURBATION THEORY}

As a final example to demonstrate the use of canonical transformations, we will consider a nonlinear perturbation of an integrable Hamiltonian system. The problem will be presented in a general form here to explain the concept and methods. Chapter 6 will cover a nonlinear perturbation of the third order while Chapter 7 will discuss an isolated nonlinear perturbation of any order.

Suppose that the problem can be described by a Hamiltonian

$$
H=H_{0}(J)+F(\phi, J, \theta)
$$

where $H$ has been written in terms of action-angle variables of the unperturbed problem. The perturbing term $F(\phi, J, \theta)$ contains nonlinear terms and is 
dependent on the independent variable $\theta$. Furthermore, $F$ is a periodic function of $\theta$ and $\phi$ and has zero average with respect to them, i.e.,

$$
\int_{0}^{2 \pi} d \theta \int_{0}^{2 \pi} d \phi F(\phi, J, \theta)=0
$$

If $F$ has a nonzero average, the average value of $F$ can be absorbed into $H_{0}(J)$.

Since $F$ is considered a perturbation to a known system, we will use a perturbation theory to study its effect. To do this we will perform a canonical transformation to a new set of variables. We would like the new Hamiltonian to depend only on the new momenta. If we succeed in this, the problem is solved since the new momenta are constants of the motion, and we have found the surface in phase space to which the motion is confined. On the other hand we cannot hope to succeed in one step. Therefore we will attempt to do this perturbatively. That is, we do not demand that the Hamiltonian be completely independent of the new coordinates and the time, but rather only approximately so. Thus we seek a transformation which replaces the perturbing term $F$ with a new perturbing term $F^{\prime}$ which is of higher order. If we imagine that $F$ is of order $\epsilon$, then $F^{\prime}$ should be of order $\epsilon^{2}$. To achieve this let us select a generating function of the second type for a transformation from $(J, \phi)$ to $\left(J_{1}, \phi_{1}\right)$ of the following form:

$$
F_{2}\left(\phi, J_{1}, \theta\right)=\phi J_{1}+G\left(\phi, J_{1}, \theta\right)
$$

The above transformation is close to the identity provided that $G$ is small. The new coordinates and Hamiltonian are given by

$$
\begin{aligned}
\phi_{1} & =\phi+G_{J_{1}} \\
J & =J_{1}+G_{\phi} \\
H_{1} & =H+G_{\theta}
\end{aligned}
$$

where the subscripts indicate partial differentiation.

The task is now to find a $G$ which eliminates the perturbing term to first order. We do this by examining the new Hamiltonian after substituting the transformed variables:

$$
H=H_{0}\left(J_{1}+G_{\phi}\right)+F\left(\phi, J_{1}+G_{\phi}, \theta\right)+G_{\theta}
$$


Note that we have substituted so that the Hamiltonian is a function of the same variables as $G$, the old coordinates and the new momenta. Eventually we must complete the substitution; however, at this time it is more convenient to work with the mixed variables. Equation (5.5) can be rewritten in the interesting form

$$
\begin{aligned}
H= & H_{0}\left(J_{1}\right)+\left[H_{0}\left(J_{1}+G_{\phi}\right)-H_{0}\left(J_{1}\right)-\nu\left(J_{1}\right) G_{\phi}\right] \\
& +\left[F\left(\phi, J_{1}+G_{\phi}, \theta\right)-F\left(\phi, J_{1}, \theta\right)\right] \\
& +\nu\left(J_{1}\right) G_{\phi}+G_{\theta}+F\left(\phi, J_{1}, \theta\right),
\end{aligned}
$$

where $\nu\left(J_{1}\right)$ is the frequency as a function of amplitude of the unperturbed problem. If we can find a solution to the equation

$$
\nu\left(J_{1}\right) G_{\phi}+G_{\theta}+F\left(\phi, J_{1}, \theta\right)=0,
$$

$G$ will be a quantity of order $F$. All other parts of the new Hamiltonian are either independent of the coordinates and time or are of order $F^{2}$. To see this more easily we can expand for small $G$ to obtain

$$
H=H_{0}\left(J_{1}\right)+\left[\nu_{J_{1}} G_{\phi}^{2} / 2+F_{J_{1}} G_{\phi}\right]+\nu\left(J_{1}\right) G_{\phi}+G_{\theta}+F\left(\phi, J_{1}, \theta\right) .
$$

We must find the periodic solution to Eq. (5.7). The periodicity requirement is obvious for the angle variables. The solution must also be periodic in $\theta$ so that $G$ does not grow in $\theta$ and thus destroy the small approximation. In order for a periodic solution to exist to Eq. (5.7), it is necessary that the average value of $F$ vanish. This was anticipated by our earlier requirement in Eq. (5.2).

Since both $F$ and $G$ are periodic functions of $\phi$, they can be Fourier analyzed.

$$
\begin{aligned}
& F\left(\phi, J_{1}, \theta\right)=\sum_{m} f_{m}\left(J_{1}, \theta\right) e^{i m \phi} \\
& G\left(\phi, J_{1}, \theta\right)=\sum_{m} g_{m}\left(J_{1}, \theta\right) e^{i m \phi} .
\end{aligned}
$$

Then the equation to be solved for $G$ becomes

$$
\left[i m \nu\left(J_{1}\right)+\frac{\partial}{\partial \theta}\right] g_{m}=-f_{m} \text {. }
$$

Exercise: Show that the periodic solution of Eq. (5.10) is

$$
g_{m}=\frac{i}{2 \sin \pi m \nu} \int_{\theta}^{\theta+2 \pi} e^{i m \nu\left(\theta^{\prime}-\theta-\pi\right)} f_{m}\left(\theta^{\prime}\right) d \theta^{\prime}
$$


Finally the full expression for $G$ is given by

$$
G=\sum_{m} \frac{i}{2 \sin \pi m \nu} \int_{\theta}^{\theta+2 \pi} d \theta^{\prime} f_{m}\left(\theta^{\prime}\right) e^{i m\left[\phi+\nu\left(\theta^{\prime}-\theta-\pi\right)\right]} .
$$

Sometimes it is desirable to make use of the fact that $F$ is a periodic function of $\theta$ and $\phi$ to expand it as a double Fourier series

$$
F=\sum_{m, n} f_{m n}\left(J_{1}\right) e^{i(m \phi-n \theta)} .
$$

Therefore, the solution to Eq. (5.10) can also be written

$$
G=i \sum_{m, n} \frac{f_{m n}\left(J_{1}\right) e^{i(m \phi-n \theta)}}{m \nu-n} .
$$

Recall that our original purpose was to transform the Hamiltonian into a form which is independent of the coordinates and the time. Now the new Hamiltonian in Eq. (5.8) becomes

$$
\begin{aligned}
H_{1} & =H_{0}\left(J_{1}\right)+\left[F_{J_{1}} G_{\phi}+\nu_{J_{1}} G_{\phi}^{2} / 2\right]+\cdots \\
& \equiv H_{0}\left(J_{1}\right)+F^{\prime}\left(J_{1}, \phi_{1}, \theta\right)
\end{aligned}
$$

The remaining nonlinear term can be separated into a part which depends only on the new action variable and into another part which involves $J_{1}, \phi_{1}$ and $\theta$ but which has zero average value. This oscillatory term is the object of the next canonical transformation, whereas the term which is a function of the new action variable $J_{1}$ leads to a change of frequencies with amplitude. The latter term is given by

$$
\bar{F}^{\prime}=\frac{1}{4 \pi^{2}} \int_{0}^{2 \pi} d \theta \int_{0}^{2 \pi} d \phi\left[F_{J_{1}} G_{\phi}+\nu_{J_{1}} G_{\phi}^{2} / 2\right] .
$$

Finally the new Hamiltonian can be written

$$
\begin{aligned}
H_{1} & =\underbrace{\left[H_{0}\left(J_{1}\right)+\bar{F}^{\prime}\left(J_{1}\right)\right]}_{H_{01}\left(J_{1}\right)}+\underbrace{\left[F^{\prime}-\bar{F}^{\prime}\right]} \\
& \equiv F_{1}\left(\phi_{1}, J_{1}, \theta\right)
\end{aligned}
$$

and the new frequency becomes

$$
\nu_{1}\left(J_{1}\right)=\frac{\partial H_{01}}{\partial J_{1}}=\nu\left(J_{1}\right)+\frac{\partial \bar{F}^{\prime}}{\partial J_{1}}
$$


Note that if we examine the new perturbing term $F_{1}$, it is second order in the strength of the perturbation. In addition it is higher order in $J_{1}$. If the original perturbation has a lowest-order contribution of order $J^{b}$, then the new term is of order $J_{1}^{(2 b-1)}$. Therefore, for sufficiently small $J_{1}$, we can neglect $F_{1}$. If this is done, we have a new Hamiltonian which depends only upon the new momenta. Therefore the new momenta are constants of the motion and can be used to label curves in phase space.

To proceed to higher order in perturbation theory there are two approaches. In the first approach we return to the generating function in Eq. (5.3) and express it as a power series in the strength of the perturbation. Then upon substitution into the Hamiltonian, we obtain a hierarchy of equations as we cancel the perturbing terms order by order. In this approach if $\epsilon$ is the strength of the perturbing term, after the $n^{\text {th }}$ step we are left with a perturbing term of order $\epsilon^{(n+1)}$.

In the second approach we begin where we left off and make successive canonical transformations which are formally identical to the first one. This method is called superconvergent perturbation theory and was first introduced in this context by Kolmogorov in his proof of the KAM theorem. ${ }^{4 a}$ It is called superconvergent because on the $n^{\text {th }}$ step the remaining perturbing term is of order $\epsilon^{2^{n}}$. However, the name does not imply that the method converges! If the procedure does converge, then it does so much faster than the first method. Unfortunately these methods do not always work; it is these divergent cases that are called nonintegrable.

Everything would be fine if $G$ were always small; however, a quick inspection of Eq. (5.14) shows that this is not the case for arbitrary $\nu$. There are resonances whenever

$$
\nu=m / n, m, n \text { integers . }
$$

This happens because we have required periodic solutions to the equation for $G$. It is straightforward to see that if the resonance condition is satisfied, there are no periodic solutions to Eq. (5.10). In fact the amplitude of the solution grows linearly in $\theta$.

But remember that for a nonlinear problem the frequency $\nu$ is generally a function of amplitude. So that if a resonance causes growth, this changes the tune and the system moves off resonance. Perhaps the infinities are a deficiency of the approach. In the vicinity of a resonance, another approach is in order.

To study the neighborhood of a resonance let the frequency $\nu$ be close to a single resonance and assume that all other resonances can be neglected, the Hamiltonian then takes the form

$$
H=H_{0}(J)+f_{m n} \cos (m \phi-n \theta) .
$$


In this case it is possible to find an exact integral of the motion by making a transformation to a coordinate system which rotates in phase space. This is accomplished with the generating function $F$ from $(\phi, J)$ to $(\psi, K)$, given by

$$
F_{2}(\phi, K, \theta)=\left(\phi-\frac{n}{m} \theta\right) K
$$

so that

$$
\psi=\phi-\frac{n}{m} \theta, \quad J=K
$$

and

$$
H_{1}=H_{0}(K)-\frac{n}{m} K+f_{m n} \cos (m \psi) .
$$

Since $H_{1}$ is now independent of $\theta$, it is a constant of the motion and can be used to label particle trajectories in phase space.

In the next section we will illustrate the techniques just discussed in the specific example of a cubic resonance.

\section{ONE-DIMENSIONAL CUBIC NONLINEAR RESONANCES}

In the last section of Chapter 5 we showed how to use canonical transformations to get rid of oscillatory terms in the Hamiltonian. In this chapter we will build on what we have already learned and apply it to a system with third-order resonance and study the phase space behavior near resonance in detail.

\subsection{The Behavior far from Resonance}

Consider a particle moving in a circular accelerator or a storage ring in the presence of a sextupole field. In this case the Hamiltonian is

$$
H=\frac{1}{2}\left(p^{2}+K x^{2}\right)+\epsilon x^{3} f(s) .
$$

Here we use $s$ as the independent variable. Recall that $f$ is periodic with period $C$ (the circumference) in $s$. In addition $f$ may have stronger periodicity imposed by design. Transforming to the action-angle variables introduced in Section 4.3

$$
\begin{aligned}
& x=\sqrt{2 J \beta} \cos \phi \\
& p=-\sqrt{2 J / \beta} \sin \phi
\end{aligned}
$$

we obtain the new Hamiltonian:

$$
\begin{aligned}
H & =J / \beta+\epsilon \sqrt{8}(J \beta)^{3 / 2} \cos ^{3} \phi f(s) \\
& \equiv J / \beta(s)+V(\phi, J, s)
\end{aligned}
$$


Our goal is to construct a canonical transformation so that the new Hamiltonian or the new action is an approximate constant of motion. Following the outline in Chapter 5 , we propose a generating function $F_{2}$ given by

$$
F_{2}\left(\phi, J_{1}, s\right)=\phi J_{1},+G\left(\phi, J_{1}, s\right)
$$

Then the canonical transformation generated by $F_{2}$ yields

$$
\begin{aligned}
\phi_{1} & =\phi+G_{J_{1}}, \\
J & =J_{1}+G_{\phi}, \\
H_{1} & =H+G_{s} .
\end{aligned}
$$

We now try to find the function $G$ such that

$$
\frac{1}{\beta(s)} G_{\phi}+G_{s}=-V\left(\phi, J_{1}, s\right) \text {. }
$$

From Eq. (6.3) we know that the perturbing term is

$$
V\left(\phi, J_{1}, s\right)=\frac{\epsilon}{\sqrt{2}}\left(J_{1} \beta(s)\right)^{3 / 2} f(s)[\cos 3 \phi+3 \cos \phi] .
$$

Then we can solve Eq. (6.6) with the method used for Eq. (5.7) to obtain

$$
\begin{aligned}
G= & -\frac{\epsilon}{\sqrt{2}}\left(J_{1}\right)^{3 / 2}\left\{\frac{3}{2 \sin \pi \nu} \int_{s}^{s+C} d s^{\prime} f\left(s^{\prime}\right) \beta\left(s^{\prime}\right)^{3 / 2} \sin \left[\phi+\psi\left(s^{\prime}\right)-\psi(s)-\pi \nu\right]\right. \\
& \left.+\frac{1}{2 \sin 3 \pi \nu} \int_{s}^{s+C} d s^{\prime} f\left(s^{\prime}\right) \beta\left(s^{\prime}\right)^{3 / 2} \sin 3\left[\phi+\psi\left(s^{\prime}\right)-\psi(s)-\pi \nu\right]\right\}
\end{aligned}
$$

Since the phase of betatron motion does not advance uniformly like a harmonic oscillator, the factor of $\nu \theta$ in Eq. (5.12) is replaced in Eq. (6.8) by $\psi(s)$ where

$$
\psi(s) \equiv \int_{0}^{s} \frac{d s^{\prime}}{\beta\left(s^{\prime}\right)}
$$


Next we have to evaluate the average of the new perturbing term in Eq. (5.16). $V_{J_{1}}$ and $G_{\phi}$ are given by

$$
\begin{gathered}
V_{J_{1}}=\frac{\partial V}{\partial J_{1}}=\frac{3}{2} \frac{\epsilon}{\sqrt{2}}\left(J_{1}\right)^{1 / 2} \beta(s)^{3 / 2} f(s)[\cos 3 \phi+3 \cos \phi] \\
G_{\phi}=-\frac{\epsilon}{\sqrt{2}}\left(J_{1}\right)^{3 / 2}\left\{\frac{3}{2 \sin \pi \nu} \int_{s}^{s+C} d s^{\prime} f\left(s^{\prime}\right) \beta\left(s^{\prime}\right)^{3 / 2} \cos \left[\phi+\psi\left(s^{\prime}\right)-\psi(s)-\pi \nu\right]\right. \\
\left.+\frac{3}{2 \sin 3 \pi \nu} \int_{s}^{s+C} d s^{\prime} f\left(s^{\prime}\right) \beta\left(s^{\prime}\right)^{3 / 2} \cos 3\left[\phi+\psi\left(s^{\prime}\right)-\psi(s)-\pi \nu\right]\right\} \cdot(6.10)
\end{gathered}
$$

First we average over $\phi$ to get rid of the cross term and then average over $s$ to obtain

$$
\begin{aligned}
& \left\langle V_{J_{1}} G_{\phi}\right\rangle^{s, \phi}=-\frac{9 \epsilon^{2}}{16}\left(J_{1}\right)^{2} \int_{0}^{C} d s \beta(s)^{3 / 2} f(s) \int_{s}^{s+C} \beta\left(s^{\prime}\right)^{3 / 2} f\left(s^{\prime}\right) d s^{\prime} \\
& \times\left\{\frac{3 \cos \left(\psi\left(s^{\prime}\right)-\psi(s)-\pi \nu\right)}{\sin \pi \nu}+\frac{\cos 3\left(\psi\left(s^{\prime}\right)-\psi(s)-\pi \nu\right)}{\sin 3 \pi \nu}\right\} .
\end{aligned}
$$

If the actual distribution of sextupoles is known, the integral in Eq. (6.11) can be evaluated. If we drop the fluctuating term, the new Hamiltonian is given by

$$
\bar{H}=H_{0}\left(J_{1}\right)+\left\langle G_{\phi} V_{J_{1}}\right\rangle .
$$

Recall that the oscillation frequency is obtained by taking the derivative of the Hamiltonian with respect to $K$. This implies that there is an amplitude dependence of the frequency due to the second-order effect of the sextupole perturbation;

$$
\nu\left(J_{1}\right)=\nu+\frac{\partial}{\partial J_{1}}\left\langle G_{\phi} V_{J_{1}}\right\rangle .
$$

Note that the additional term in the new Hamiltonian in Eq. (6.11) is of order $J^{2}$, and thus the tune in Eq. (6.12) varies linearly with $J$. This is similar to the first-order effect of an octupole perturbation $\left(\sim x^{4}\right)$; therefore, a sextupole perturbation in second order produces an octupole-like nonlinear frequency shift with amplitude. This is only true, however, if the tune is far from resonance. 


\subsection{The THIRD INTEger Resonance}

The approach we used so far will work fine if the perturbing terms are small. However, this is not the case whenever $\nu$ is close to an integer or a third integer, because then the denominator in Eq. (6.8) will become very small, and the entire procedure breaks down. In such a situation we can solve the problem when the tune is close to a particular resonance as indicated in the previous chapter. For example, let us consider the case when $\nu$ is very close to a third integer,

$$
\nu=\frac{n_{0}}{3}+\delta \text {. }
$$

It is possible to find an integral of the motion for the Hamiltonian by going into a rotating coordinate system provided that we can neglect other resonances. Let us return to the Hamiltonian in Eq. (6.3),

$$
H=J / \beta(s)+\epsilon \sqrt{8} \beta^{3 / 2} J^{3 / 2} f(s) \cos ^{3} \phi .
$$

In this section it is convenient to use the coordinate system developed in Eq. (4.58) in which the phase advances uniformly.

In this case the transformation yields

$$
\begin{aligned}
& \phi_{1}=\phi-\left[\int_{0}^{s} \frac{d s^{\prime}}{\beta(s)}-\frac{\nu s}{R}\right] \equiv \phi-\chi(s) \\
& J_{1}=J .
\end{aligned}
$$

The function $\chi(s)$ is a known function of $s$ calculated from the known $\beta(s)$. The action variable is unchanged while the Hamiltonian becomes

$$
H_{1}=\frac{\nu J_{1}}{R}+\epsilon \sqrt{8} \beta(s)^{3 / 2} J_{1}^{3 / 2} f(s) \cos ^{3}\left(\phi_{1}+\chi(s)\right)
$$

Since the perturbed part of the Hamiltonian is a periodic function of $\phi_{1}$ and $s$, it can be written as a Fourier series:

$$
H_{1}=\frac{\nu J_{1}}{R}+J_{1}^{3 / 2} \sum_{m, n} A_{m n} \cos \left(m \phi_{1}-n s / R+\alpha_{m n}\right) .
$$

The Fourier series is finite in $\phi_{1}$ and (possibly) infinite in $s / R$. To consider the neighborhood of the third integer resonance we drop all terms except the resonance term to find

$$
H_{1} \simeq \frac{\nu J_{1}}{R}+J_{1}^{3 / 2} A_{3 n_{0}} \cos \left(3 \phi_{1}-\frac{n_{0} s}{R}+\alpha_{3 n_{0}}\right)
$$

The other terms can be moved to higher order by a canonical transformation as demonstrated earlier in this section. To simplify the following discussion we set $\alpha_{3 n_{0}}$ equal to zero. 
Now consider another canonical transformation to new variables $(\psi, K)$ given by

$$
F_{2}=K\left(\phi_{1}-\frac{n_{0}}{3} \theta\right)
$$

then

$$
\begin{aligned}
\psi & =\phi_{1}-\frac{n_{0}}{3} \theta \\
J_{1} & =K
\end{aligned}
$$

This yields the Hamiltonian

$$
H_{1} \simeq \delta K+K^{3 / 2} A_{3 n_{0}} \cos (3 \psi) .
$$

\subsection{Phase Space Structure}

Now we finally have a Hamiltonian independent of the "time" variable $s$. It is now straightforward to find out the behavior of the particle by examining the phase-space structure of the system under various conditions. For example, the first thing to find are the fixed points of the motion. These can be found by solving

$$
\frac{\partial H_{1}}{\partial K}=0 \quad, \quad \frac{\partial H_{1}}{\partial \psi}=0
$$

which is given explicitly by

$$
\begin{aligned}
\delta+\frac{3}{2} A_{3 n_{0}} K^{1 / 2} \cos 3 \psi & =0 \\
\sin 3 \psi & =0 .
\end{aligned}
$$

The solutions to Eq. (6.24) are

$$
\begin{aligned}
\psi & =\pi / 3 \quad, 3 \pi / 3 \quad, \quad 5 \pi / 3 \\
K_{r} & =\left(\frac{2 \delta}{3 A_{3 n_{0}}}\right)^{2} .
\end{aligned}
$$

These fixed points are shown in Fig. 6.1. Note that if we transformed back to $\left(J_{1}, \phi_{1}\right)$ variables the figure would rotate in phase space. 
It is clear that the presence of the perturbation divides phase space into disjoint regions. When the tune is away from resonance and the amplitude is small, the unperturbed term dominates and the trajectory is a circle around the origin representing stable unperturbed motion. When the amplitude gets larger, the perturbing term makes its presence known by deforming the trajectory into a triangular shape, but the motion is still stable. If the amplitude now grows larger than $K_{r}$, the trajectory ceases to form a closed curve. This leads to unstable motion of the particle.

Note that Eq. (6.25) indicates that the amplitude of the fixed points will shrink to zero when

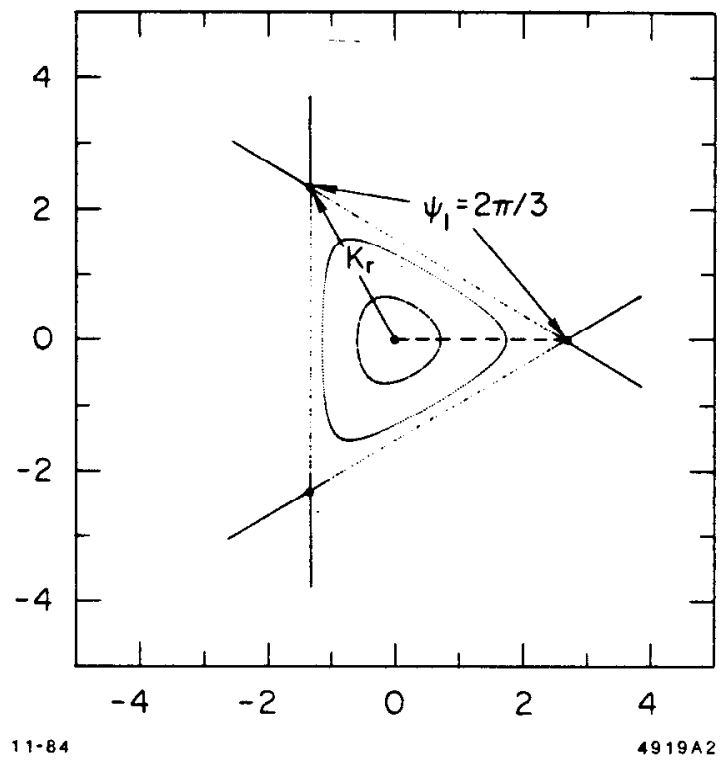

Fig. 6.1. Phase space structure of third order resonance. the tune is right on resonance $(\delta=0)$, and the stable fixed point at the origin becomes an unstable fixed point.

Although most designers of accelerators try very hard to avoid resonances, the third-integer resonance has actually been used by design to slowly extract a beam from a synchrotron for high energy experimental programs. This process, called resonant extraction, makes use of the large amplitude of the particles moving out on a separatrix to separate them from the core of the beam and deflect them out of the accelerator. For further details of this process refer to Ref. 13.

\subsection{Stopband WidTh OF THE THIRD-ORDER RESONANCE}

Assuming that the amplitude at which the resonance occurs is already known as $K_{r}$, then the tune separation needed to be away from unstable motion is

$$
\delta>\frac{3 A_{3 n_{0}}}{2} K_{r}^{1 / 2} \text {. }
$$

The term 'stopband width' is defined to indicate this separation, i.e.,

$$
\text { Stopband width } \Delta \nu=\frac{3 A_{3 n_{0}}}{2} K_{r}^{1 / 2} \text {. }
$$


To relate all of this to the actual physical world of a beam distribution, we must recall from Eq. (4.57) that the emittance of a beam is related to the action variable $K$,

$$
\epsilon=\langle K\rangle \text {. }
$$

Therefore, for a beam with emittance $\epsilon$, the third-order stopband width is

$$
\Delta \nu=\frac{3 A_{3 n_{0}}}{2} \sqrt{\epsilon} .
$$

\section{A ONE-DIMENSIONAL ISOLATED NONLINEAR RESONANCE}

Thus, we have seen how to apply the canonical transformation method to solve a third-order nonlinear perturbation to an otherwise linear Hamiltonian system. Now we want to extend this technique to a system subjected to a nonlinear perturbation of any order to understand the behavior of the system as long as each resonance acts independently. A system under the action of isolatē nonlinear perturbation generated by a nonlinear term $\sim x^{k}$ can be described by the Hamiltonian

$$
H=\nu I+\alpha(I)+\epsilon I^{k / 2} \cos (m \phi-n \theta) .
$$

Thus, we suppose that we are close to a particular resonance and that all other resonances can be neglected. We make a canonical transformation to a rotating system in phase space with the generating function:

$$
F_{2}=(\phi-n / m \theta) I_{1}
$$

then

$$
\begin{gathered}
\psi=\phi-n / m \theta \quad, \quad I_{1}=I \\
H_{1}=H-n / m I_{1}=\delta I_{1}+\alpha\left(I_{1}\right)+\epsilon I^{k / 2} \cos (m \psi)
\end{gathered}
$$

where

$$
\delta=\nu-n / m .
$$

The Hamiltonian has been successfully cast in a form explicitly independent of the "time" variable $\theta$; thus, it is a constant of the motion. 


\subsection{FIXED POINTS}

In the phase space $\left(\psi, I_{1}\right)$ we can find a set of points where the trajectories are stationary. Those fixed points can be obtained by the conditions:

$$
\frac{\partial H_{1}}{\partial I_{1}}=\frac{\partial H_{1}}{\partial \psi}=0
$$

which implies

$$
\begin{aligned}
& \sin m \psi_{0}=0 \\
& \delta+\alpha^{\prime}\left(I_{1}\right)+\cos \left(m \psi_{0}\right) \frac{k}{2} \in I_{1}^{k / 2-1}=0 .
\end{aligned}
$$

In the polar coordinates $\left(\psi, I_{1}\right)$, these are a string of points surrounding the origin, as shown in Fig. 7.1.

In fact when $\sin m \psi_{0}=0, \cos m \psi_{0}=$ \pm 1 and for different signs of $\cos m \psi$ the characteristics of the fixed points are different. The trajectories surrounding stable fixed points, SFP, are closed (either circles or ellipses), while those surrounding unstable fixed points, UFP, are open (hyperbolic). It will be shown in the next section that those angles corresponding to $\cos m \psi_{0}=1$ are unstable fixed points while those with $\cos m \psi_{0}$ $=-1$ are stable fixed points.

Suppose we define $I_{r}$ as that amplitude which yields an oscillation frequency at resonance, i.e.,

$$
\nu+\alpha^{\prime}\left(I_{r}\right)=n / m
$$

then Eq. (7.6) becomes

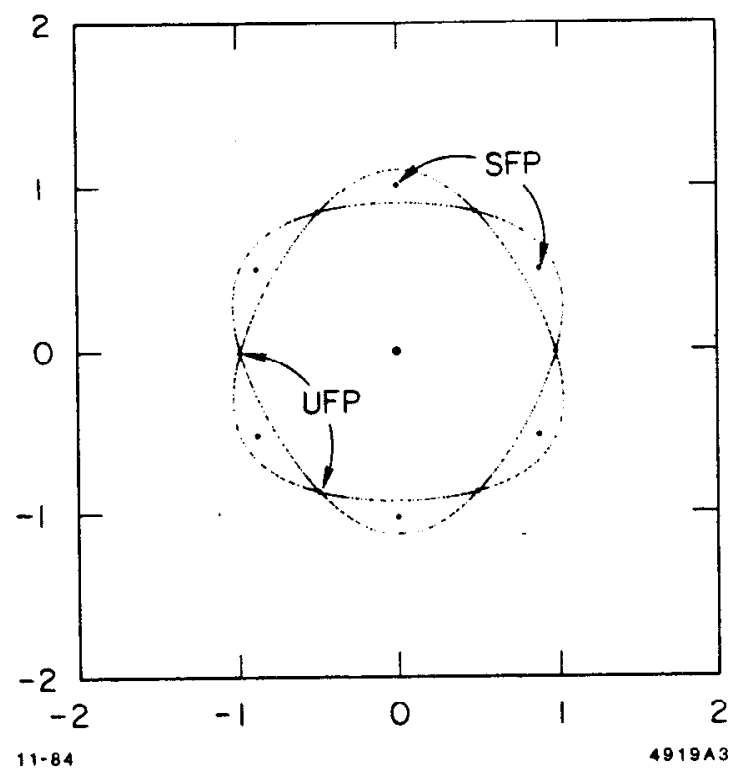

Fig. 7.1. A string of fixed points in the $(\psi, I)$ phase space. Shown is the sixth order perturbation. 


$$
\begin{aligned}
& \alpha^{\prime}\left(I_{1}\right)-\alpha^{\prime}\left(I_{r}\right) \\
& \quad+\cos \left(m \psi_{0}\right) \frac{k}{2} \epsilon I_{1}^{k / 2-1}=0
\end{aligned}
$$

or expanding for $I_{1}$ close to $I_{r}$

$$
\begin{aligned}
& \left(I_{1}-I_{r}\right)= \\
& -\frac{k}{2} \frac{\epsilon}{\alpha^{\prime \prime}} I_{r}^{k / 2-1} \cos \left(m \psi_{0}\right) .
\end{aligned}
$$

Therefore, the amplitude of UFP is slightly less than $I_{r}$ while the amplitude of SFP is slightly larger than $I_{r}$. In summary, the phase space structure close to a particular resonance is a string of stable islands at a particular amplitude determined by the tune and nonlinear detuning of the system, as shown in Fig. 7.2.

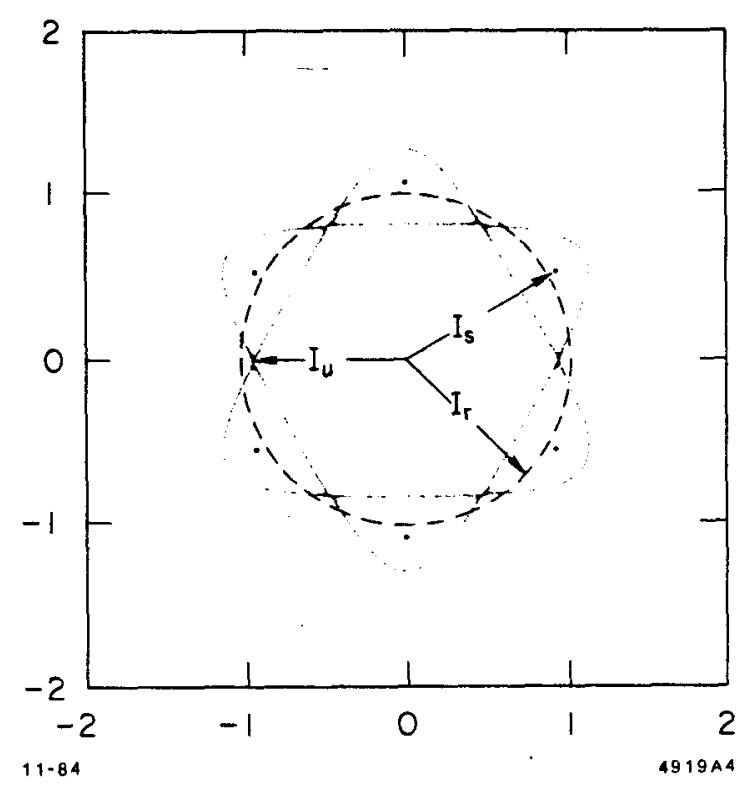

Fig. 7.2. Relative locations of stable and unstable fixed points $\left(I_{u}<I_{r}<I_{s}\right)$.

\subsection{RESONANCE ISLAND WIDTH}

The boundaries of the stable islands are formed by curves joining the unstable fixed points. They are called separatrices and their equation can be easily found by the fact that the Hamiltonian is a constant on the curve.

From Eqs. (7.3) and (7.7), we have

$$
\begin{aligned}
\delta I+\alpha(I)+\epsilon I^{k / 2} \cos m \psi & =\delta I_{u}+\alpha\left(I_{u}\right)+\epsilon I_{u}^{k / 2} \\
\alpha(I)-I \alpha^{\prime}\left(I_{r}\right)+\epsilon I^{k / 2} \cos m \psi & =\alpha\left(I_{u}\right)-I_{u} \alpha^{\prime}\left(I_{r}\right)+\epsilon I_{u}^{k / 2} .
\end{aligned}
$$

where $I_{u}$ is the action at the unstable fixed point. Expanding for $I$-close to $I_{u}$ gives the difference of the amplitude between $I$ and $I_{u}$,

$$
\left(I-I_{u}\right)^{2} \simeq \frac{2 \epsilon I_{u}^{k / 2}(1-\cos m \psi)}{\alpha^{\prime \prime}} .
$$

From Eq. (7.11) we can find the maximum separation or island width

$$
\Delta I=2 \sqrt{\frac{\epsilon I_{u}^{k / 2}}{\alpha^{\prime \prime}}} .
$$


So far we have worked with a Hamiltonian with a general amplitude dependence of the tune. Next let us keep only the lowest-order term, which is quadratic in $I$ and work out some of the properties of the island more simply. When the detuning term is quadratic in $I$, the Hamiltonian (7.3) becomes

$$
H=\delta I+\frac{1}{2} a I^{2}+\epsilon I^{k / 2} \cos m \psi
$$

and the definition for $I_{r}$ becomes

$$
\nu+a I_{r}=n / m .
$$

In order to see the Hamiltonian in the vicinity of $I_{r}$, we express the Hamiltonian in terms of $p$, where $I=p+I_{r}$,

$$
H=\frac{1}{2} a p^{2}+\epsilon I_{r}^{k / 2} \cos m \psi
$$

where constant terms have been dropped. Again it is easy to see the island width using Hamiltonian at the UFP to obtain

$$
\frac{1}{2} a p_{\max }^{2}-\epsilon I_{r}^{k / 2}=\epsilon I_{r}^{k / 2}
$$

Therefore the island width is given by

$$
\Delta I=2 \sqrt{\frac{\epsilon}{a} I_{r}^{k / 2}} .
$$

Keep in mind that this is only valid when $I-I_{r}<<I_{r}$. Another condition to be met is that other resonances should be far away. If the widths calculated using isolated resonances are such that they overlap each other, then it is clearly incorrect to consider the resonances isolated.

\subsection{ISLAND SEPARATION}

To find the distance to the next resonance, we will first find the spacing in frequency and then convert that to amplitude. Recall that the linear amplitude dependence of the tune gives

$$
\Delta I=\Delta \nu / a .
$$

In frequency space neighboring primary resonances occur at $n \pm 1, m \pm 1$; therefore, the spacing is given by

$$
\Delta I=\frac{\Delta \nu}{a}=\frac{\Delta(n / m)}{a} \simeq \frac{1}{m a} \text { or } \frac{n}{m^{2} a} .
$$


Using the smaller spacing, we find that the condition for spacing to be larger than island width is

$$
a<<\frac{n}{m^{2} \epsilon I_{r}^{k / 2}}
$$

Recall that Eq. (7.17) requires that $I-I_{r}<<I_{r}$, giving

$$
\epsilon / a I_{r}^{k / 2} \ll I_{r}^{2} / 4
$$

or

$$
a>>\frac{4 \epsilon I_{r}^{k / 2}}{I_{r}^{2}} .
$$

Equations (7.20) and (7.22) set a limit to the validity of our analysis. These two conditions require that the nonlinear detuning, $a$, must be moderate. It should be neither too small (since then the islands do not close) nor too large (since then the resonances do not separate).

\section{$7^{-4}$ The Pendulum Equation}

Having understood the phase space structure in general, we are ready to zoom into a particular island as suggested by Eq. (7.15),

$$
H=\frac{1}{2} a p^{2}+\epsilon I_{r}^{k / 2} \cos m \psi .
$$

In this form we are considering the trajectories close to a particular resonance $I_{r}$.

From Hamilton's equations the equations of motion in $(\psi, I)$ coordinates are

$$
\begin{aligned}
& \dot{\psi}=\frac{\partial H}{\partial p}=a p \\
& \dot{p}=-\frac{\partial H}{\partial \psi}=-\epsilon m I_{r}^{k / 2} \sin m \psi .
\end{aligned}
$$

Combining the two equations gives

$$
\bar{\psi}+a \epsilon m I_{r}^{k / 2} \sin m \psi=0 .
$$

This is the equation of motion for a pendulum with familiar phase space structure shown in Fig. 7.3. 
When the amplitude is small, a small amplitude oscillation frequency $\Omega$ can be obtained from (7.24) by approximating

$$
\sin m \psi \simeq m \psi
$$

This yields

$$
\Omega^{2}=a \in I_{r}^{k / 2} m^{2} .
$$

From this frequency an alternate expression for the overlap condition can be derived. The frequency of the neglected terms should be large compared to $\Omega$. In other words, since the lowest frequency of the neglected terms is simply unity, we find

$$
\sqrt{a \in I_{r}^{k / 2} m^{2}}<<1
$$

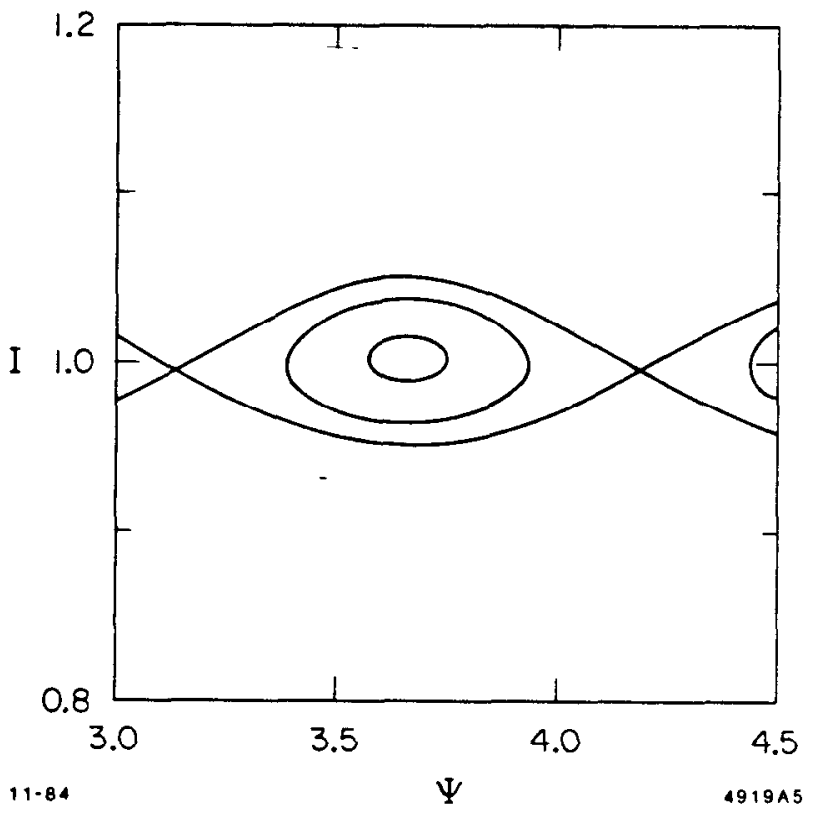

Fig. 7.3. Pendulum-like phase space structure in the vicinity of a stable fixed point.

In Section 6.1 we promised to show the way to identify stable or unstable fixed points. This can be done by looking into the neighborhood of the fixed points. It has already been shown that the fixed points occur around $\cos (m \psi)=$ \pm 1 ; we can expand the angle variable around those points to obtain

$$
\cos (m \psi) \simeq-1+\frac{m^{2} \Delta \psi^{2}}{a}
$$

in the vicinity of $\cos (q \psi)=-1$, and similarly in the vicinity of $\cos (q \psi)=1$ we have

$$
\cos (m \psi) \simeq 1-\frac{m^{2} \Delta \psi^{2}}{2}
$$

The Hamiltonian now takes the form

$$
H=\frac{1}{2} a p^{2} \pm\left(\frac{\epsilon m^{2} I_{r}^{k / 2}}{2}\right) \Delta \psi^{2}
$$

where the constant term of 1 has been dropped. It is clear now that the trajectory near $m \psi=\pi$ is elliptical and hence stable, while the trajectory near $m \psi=2 \pi$, is hyperbolic and hence unstable. 


\subsection{The Distinction of Resonances 3, 4, 5 or Higher}

There is a qualitative difference between resonances of order three or four and those of order five or higher. Third-order resonances have unstable fixed points at $J=0$ when the frequency is on resonance $(\delta=0)$. This does not necessarily imply global instability since in many cases the separatrices are bent back by the nonlinear detuning at large amplitudes. Fourth-order resonances may have stable or unstable fixed points at the origin, and fifth- or higher-order resonances have stable fixed points at the origin.

This is due to the relative powers of the action variable $I$ in the perturbation term and in the nonlinear detuning term. For third-order the perturbation dominates at small amplitude due to the $I^{3 / 2}$ dependence. For fifth- or higherorder the nonlinear detuning dominates with its $I^{2}$ dependence. The fourthorder resonance is the transition. In this case stability depends upon the relative size of the coefficients in the two terms.

To see this effect in the fourth order resonance consider the Hamiltonian exactly at resonance:

$$
H=\frac{1}{2} a I^{2}+\epsilon I^{2} \cos 4 \psi \equiv E
$$

which yields

$$
I^{2}=\frac{E}{\frac{1}{2} a+\epsilon \cos 4 \psi} .
$$

In order to keep the amplitude positive definite, $a$ has to be greater than $2 \epsilon$. Physically that means the nonlinear detuning has to be strong enough to bend the separatrices back to form a closed curve.

For fifth order or higher, $n>5$, the small oscillation is always stable. Thus a one-dimensional nonlinear resonance causes instability at small amplitude when it is of third- or fourth-order, but not for fifth-order or higher.

\subsection{SOLUTION ON THE SEPARATRIX}

We know that when the amplitude is small, the particles oscillate with frequency $\nu$. But as the amplitude increases and gets closer to the separatrix, the frequency decreases. Now we want to see what actually happens when the particle is sitting on the separatrix. Consider the Hamiltonian

$$
H=\frac{p^{2}}{2}-\cos \phi
$$

It has already been shown that the separatrix passes through the unstable fixed point at phase angle $-\pi$ and $\pi$; therefore the equation for the separatrix is given 
by

$$
\frac{p^{2}}{2}-\cos \phi=1
$$

which yields

$$
\dot{\phi}=p= \pm \sqrt{2(1+\cos \phi)}= \pm 2 \cos \phi / 2 \text {. }
$$

It can be shown that the solution for $\phi$ is given by

$$
\phi=4 \tan ^{-1}\left(e^{t}\right)-\pi
$$

Exercise: Prove that Eq. (7.37) is indeed the solution of Eq. (7.36).

It is clear that the rate of approach toward unstable fixed point decreases as the particle gets closer to the UFP. From Eq. (7.37) it takes an infinite amount of time to actually reach it. This makes it much more sensitive to perturbation.

The solution above is for motion on the separatrix of one isolated resonance, in this case the simple pendulum. In the general system there are many other resonances. We have argued earlier that provided the spacing of the resonances is larger than the widths of the resonances (the Chirikov criterion), ${ }^{7}$ then the isolated resonance picture is useful. However, the effects of the other resonances first become visible in the neighborhood of the separatrix.

The problem is that although the oscillations around stable fixed points are somewhat immune to the perturbations of neighboring resonances, the motion near unstable fixed points and the motion on separatrices are not. If we trace the trajectory which approaches the fixed point at $\pi$ back in time and also trace the trajectory which departs from $-\pi$ forward in time, we find completely different behavior in the case of multiple resonances than in the case of an isolated resonance.

In the case of a single resonance the two 'separatrices' join smoothly and are actually part of the same curve, as demonstrated above. In the case of multiple resonances the two 'separatrices' meet at a small angle. This leads to completely different behavior in the neighborhood of the separatrix even for very small perturbations. ${ }^{3,6,14}$ If we look in the neighborhood of a 'separatrix', rather than a curve, we see a band of apparently random motion. If the resonances overlap, this 'stochasticity' can spread to almost all of phase space. However, if the resonances are well isolated, this behavior is usually confined to the neighborhood of the 'separatrices' and unstable fixed points. 


\section{SUMMARY}

In the previous sections we have reviewed some of the basic techniques which can be used to analyze stability in nonlinear dynamical systems, particularly in circular particle accelerators. We have concentrated on one-dimensional systems in the examples in order to simply illustrate the general techniques.

We began with a review of Hamiltonian dynamics and canonical transformations. We then reviewed linear equations with periodic coefficients using the basic techniques from accelerator theory.

To handle nonlinear terms we developed a canonical perturbation theory. From this we calculated invariants and the amplitude dependence of the frequency. This led us to resonances.

We studied the cubic resonance in detail by using a rotating coordinate system in phase space. We then considered a general isolated nonlinear resonance. In this case we calculated the width of the resonance and estimated the spacing of resonances in order to use the Chirikov criterion to restrict the validity of the analysis. Finally the resonance equation was reduced to the pendulum equation, and we examined the motion on a separatrix. This brought us to the beginnings of stochastic behavior in the neighborhood of the separatrix.

It is this complex behavior in the neighborhood of the separatrix which causes the perturbation theory used here to diverge in many cases. In spite of this the methods developed here have been and are used quite successfully to study nonlinear effects in 'nearly integrable' systems. When used with caution and in conjunction with numerical work they give tremendous insight into the nature of the phase space structure and the stability of nonlinear differential equations.

\section{ACKNOWLEDGMENTS}

The authors would like to thank A. Chao, J. Herrera and M. Month for useful suggestions, Margaret Dienes for her careful editing, and Tor Raubenheimer for help with the figures.

\section{BIBLIOGRAPHY}

\section{Classical Mechanics and Dynamical Systems}

1. H. Goldstein, "Classical Mechanics," Addison-Wesley, 1950.

2. E. T. Whittaker, “Analytical Dynamics," Dover, 1944.

3. V. I. Arnold, "Mathematical Methods of Classical Mechanics," SpringerVerlag, 1978. 
4. KAM Theorem:

a. A. N. Kolmogorov, "On Conservation of Conditionally-Periodic Motions of a Small Change in Hamilton's Function," Kokl. Adad. Nauk SSSR 98:4 (1954) 525-530.

b. V. I. Arnold, "Small Denominators I. Mapping the Circle onto Itself," Izv. Akad. Nauk SSSR Ser. Mat. 25 (1961) 21-86.

c. V. I. Arnold, "Small Denominators II. Proof of a Theorem of A. N. Kolmogorov on the Preservation of Conditionally-Periodic Motions under a Small Perturbation of the Hamiltonian," Russian Math. Surveys 18:5 (1963).

d. V. I. Arnold, "Small Denominators III. Small Denominators and Problems of Stability of Motion in Classical and Celestial Mechanics, "Russian Math. Surveys 18:6 (1963).

e. J. Moser, "On Invariant Curves of Area-Preserving Mappings of an Annulus," Nachr. Akad. Wiss. Gottingen, Math. Phys. K1 IIa (1962) 1-20.

5. C. L. Siegel and J. Moser, "Lectures on Celestial Mechanics," SpringerVerlag, 1971.

6. M. V. Berry, "Regular and Irregular Motion," in 'Topics in Nonlinear Dynamics,' AIP Proc. No. 46 (1978) 16-120.

7. B. V. Chirikov, “A Universal Instability of Many-Dimensional Oscillator Systems," Phys. Rept. 52 (1979).

8. F. Vivaldi, “Weak Instability in Many-Dimensional Hamiltonian Systems," Rev. Mod. Phys. 56 (1984) 737-754.

9. J. J. Stoker, “Nonlinear Vibrations," Chap. VI, Interscience Publishers, New York, 1950.

\section{Accelerator Theory}

10. E. D. Courant and H. S. Snyder, "Theory of the Alternating-Gradient Synchrotron," Ann. of Phys. 3 (1958) 1-48.

11. A. Schoch, "Theory of Linear and Nonlinear Perturbations of Betatron Oscillations in Alternating-Gradient Synchrotrons," CERN 57-21, 1958.

12. G. Guignard, "A General Treatment of Resonances in Accelerators, "CERN 78-11, 1978.

13. K. R. Symon, "Extraction at a Third Integral Resonance, I, II, III, IV," Fermi Lab. Notes FN-130, 134, 140 and 144; 1968.

14. L. J. Laslett, “Stochasticity," Proc. Int. Conf. on High Energy Accelerators (1974) 394-401. 